\title{
Osteoinduction by Foamed and 3D-Printed Calcium Phosphate Scaffolds: Effect of Nanostructure and Pore Architecture
}

Albert Barba ${ }^{a, b, c}$, Anna Diez-Escudero ${ }^{a, b}$, Yassine Maazouz ${ }^{a, b}$, Katrin Rappe ${ }^{c}$, Montserrat Espanol, ${ }^{a, b}$ Edgar B. Montufar ${ }^{\mathrm{a}, \mathrm{b}, 1}$, Mar Bonany ${ }^{\mathrm{a}, \mathrm{b}}$, Joanna M. Sadowska ${ }^{\mathrm{a}, \mathrm{b}}$, Jordi Guillem-Marti ${ }^{\mathrm{a}, \mathrm{b}}$, Caroline Öhman-Mägi ${ }^{d}$, Cecilia Persson ${ }^{d}$, Maria-Cristina Manzanares ${ }^{\mathrm{e}}$, Jordi Franch $^{\mathrm{c}}$, Maria-Pau Ginebra ${ }^{\mathrm{a}, \mathrm{b}, \mathrm{f}^{*}}$

aBiomaterials, Biomechanics and Tissue Engineering Group, Department of Materials Science and Metallurgical Engineering, Universitat Politècnica de Catalunya, Av. Eduard Maristany 10-14, 08019 Barcelona, Spain

bBarcelona Research Center in Multiscale Science and Engineering, Universitat Politècnica de Catalunya, Av. Eduard Maristany 10-14, 08019 Barcelona, Spain

'Bone Healing Group, Small Animal Surgery Department, Veterinary School, Universitat Autònoma de Barcelona, 08193 Bellaterra (Barcelona), Spain

'Materials in Medicine Group, Division of Applied Materials Science, Department of Engineering Sciences, Uppsala University, 75121 Uppsala, Sweden

eHuman Anatomy and Embryology Unit, Department of Pathology and Experimental Therapeutics,

Universitat de Barcelona, 08907 L'Hospitalet de Llobregat (Barcelona), Spain

fInstitut for Bioengineering of Catalonia (IBEC), 08028 Barcelona, Spain

${ }^{1}$ Current affiliation: CEITEC - Central European Institute of Technology, Brno University of Technology, 61200 Brno, Czech Republic

*Corresponding author: maria.pau.ginebra@upc.edu

Keywords: Osteoinduction; 3D-printing; foaming; nanostructure; calcium phosphate 


\section{Abstract}

Some biomaterials are osteoinductive, i.e., they are able to trigger the osteogenic process by inducing the differentiation of mesenchymal stem cells to the osteogenic lineage. Although the underlying mechanism is still unclear, microporosity and specific surface area (SSA) have been identified as critical factors in the material-associated osteoinduction. However, only sintered ceramics, with a limited range of porosities and SSA, have been analysed so far. In this work we were able to extend this range to the nanoscale, through the foaming and 3D-printing of biomimetic calcium phosphates, obtaining scaffolds with controlled micro and nanoporosity together with a tailored macropore architecture. Calcium deficient hydroxyapatite (CDHA) scaffolds were evaluated after 6 and 12 weeks in an ectopic implantation canine model, and compared with two sintered ceramics, biphasic calcium phosphate and beta-tricalcium phosphate. Only foams with spherical concave macropores, and not 3D-printed scaffolds with convex prismatic macropores induced significant ectopic bone formation. Amongst them, biomimetic nanostructured CDHA produced the highest incidence of ectopic bone and accelerated bone formation when compared to conventional microstructured sintered calcium phosphates with the same macropore architecture. Moreover, they exhibited different bone formation patterns, since in CDHA foams the new ectopic bone progressively replaced the scaffold, whereas in sintered biphasic calcium phosphate scaffolds bone was deposited on the surface of the material, progressively filling the pore space. In conclusion, this study demonstrates that the high reactivity of nanostructured biomimetic CDHA, combined with a spherical concave macroporosity allows pushing the osteoinduction potential beyond the limits of microstructured calcium phosphate ceramics. 


\section{Introduction}

Some biomaterials are able to instruct cells and, by driving cell fate, they are able to modulate the regenerative potential of specific tissues. A good example is what has been called materialassociated osteoinduction, i.e., the capacity of some biomaterials to induce the differentiation of mesenchymal stem cells into the osteoblastic lineage, even in absence of exogenously applied bone morphogenetic proteins (BMPs). This property, that was discovered in the early 1990's for calcium phosphates $(\mathrm{CaP})^{1,2}$ is of paramount importance when designing synthetic bone grafts. Intrinsic osteoinduction can endorse the biomaterial with the capacity to regenerate bone even in compromised clinical situations, avoiding the detrimental side effects associated to the use of $\mathrm{BMPs}^{3,4}$. The relevance of this topic is linked to the high incidence of bone grafting procedures, around 2.2 million worldwide annually ${ }^{5}$. In this context, the development of synthetic bone substitutes with enhanced performance, able to outperform autologous bone grafts and to avoid their intrinsic drawbacks, e.g. limited availability, donor site pain and risk of infection or disease transmission ${ }^{6}$, is an urgent challenge.

Great attention has been paid in the last years to the parameters leading to biomaterial-associated osteoinduction. Some CaP ceramics have been found to exhibit an intrinsic osteoinductive capacity when implanted ectopically in several animal models ${ }^{7}$, which is associated to a higher bone healing capacity when implanted orthotopically, compared to non-osteoinductive ceramics ${ }^{8}$. It has also been reported that osteoinductive ceramics perform similarly to both autologous bone graft and rhBMP2-impregnated collagen sponge in repairing critical-size bone $\operatorname{defects}^{9}$. Although the mechanism underlying bone induction is still not fully understood, the textural properties of the material are believed to play a key role $\mathrm{e}^{10-13}$. However, most studies on osteoinduction of CaP have been performed with sintered ceramics, where the high temperature processing precludes the introduction of nanoporosity. Consequently, low specific surface areas (SSA) are obtained, normally in the range of $0.2-2 \mathrm{~m}^{2} / \mathrm{g}$. This leaves the hypothesis without a complete verification, and establishes the need to provide a full picture of the situation, by assessing the osteoinductive properties of $\mathrm{CaP}$ biomaterials with nanostructured features and higher SSA. 
The recent biomimetic routes based on the self-setting reaction of calcium phosphate cements (CPCs) have opened up new possibilities in this respect, since they allow obtaining nanostructured CaP scaffolds, with controlled micro and nanoporosity, together with a tailored architecture ${ }^{14}$. As a result of the setting reaction, an entangled network of hydroxyapatite nanocrystals is obtained at physiological temperature, which does not simply mimic the composition and morphology of the bone mineral phase better than sintered $\mathrm{CaP}$ biomaterials, but also generate a porous structure with specific nano/micro porosities, and consequently, much higher SSA than sintered ceramics.

One additional advantage of CPCs is their simplicity in processing, which makes them extremely versatile and compatible with many techniques. Nanostructured hydroxyapatite foams, with open interconnected macropores can be obtained by adding small amounts of a surfactant in the liquid phase of the CPC and applying mechanical stirring ${ }^{15,16}$. Moreover, biomimetic processing of hydroxyapatite is compatible with additive manufacturing strategies. Self-setting calcium phosphate inks have been designed ${ }^{17}$ that allow the fabrication of nanostructured hydroxyapatite scaffolds with controlled pore architecture, using 3D-microextrusion techniques, also known as robocasting ${ }^{18}$.

Taking advantage of these technologies, scaffolds with diverse pore architectures can be obtained, while preserving the specific textural properties typical of biomimetic ceramics. The possibility to tailor porosity at different levels, from the nano- to the macroscale, significantly extends the range of textures analyzed so far and allows addressing more in-depth the effect of the nanostructure on the osteoinductive properties of calcium phosphates. The aim of this study was to assess the relative importance of nanostructural features and scaffold architecture to the osteoinduction of calcium phosphate biomaterials in a canine ectopic model. Biomimetic calcium deficient hydroxyapatite (CDHA) scaffolds with different pore architectures were compared to two sintered CaP ceramics, i.e. a biphasic calcium phosphate (BCP) and beta-tricalcium phosphate ( $\beta$-TCP). 


\section{Materials and Methods}

\subsection{Calcium phosphate materials}

\subsubsection{Synthesis of alpha-tricalcium phosphate}

Alpha-tricalcium phosphate $\left(\alpha-\mathrm{Ca}_{3}\left(\mathrm{PO}_{4}\right)_{2}, \alpha-\mathrm{TCP}\right)$ was the solid phase of the cement used for the fabrication of the scaffolds. Briefly, $\alpha$-TCP was obtained by heating calcium hydrogen phosphate $\left(\mathrm{CaHPO}_{4}\right.$, Sigma-Aldrich, St. Louis, USA) and calcium carbonate $\left(\mathrm{CaCO}_{3}\right.$, Sigma-Aldrich, St. Louis, USA) at a $2: 1$ molar ratio for $15 \mathrm{~h}$ at $1400^{\circ} \mathrm{C}$ followed by quenching in air. Subsequently, the powder was milled in an agate ball mill (Pulverisette 6, Fritsch GmbB, Markt Einersheim, Germany), with 10 balls $(\mathrm{d}=30 \mathrm{~mm})$ for $60 \mathrm{~min}$ at $450 \mathrm{rpm}$ followed by a second milling for $40 \mathrm{~min}$ at $500 \mathrm{rpm}$ with 10 balls $(d=30 \mathrm{~mm})$ and a third one for $60 \mathrm{~min}$ at $500 \mathrm{rpm}$ with 100 balls $(\mathrm{d}=10 \mathrm{~mm})$, to attain a mean powder particle size of $2.8 \mu \mathrm{m}$.

\subsubsection{Preparation of foamed scaffolds}

To obtain the CDHA foams (CDHA-Foam) a solid phase consisting of $98 \mathrm{wt} \% \alpha-\mathrm{TCP}$ and $2 \mathrm{wt} \%$ of precipitated hydroxyapatite (PHA, Merck KGaA, Darmstadt, Germany) was mixed with an aqueous solution of 1 wt\% Polysorbate 80 (Tween $80^{\circledR}$, Sigma-Aldrich, St. Louis, USA) at a liquid to powder ratio of $0.65 \mathrm{~mL} / \mathrm{g}$. The mixture was foamed with a domestic food mixer for $30 \mathrm{~s}$ at $7000 \mathrm{rpm}$ and then transferred to Teflon cylindrical moulds ( $5 \mathrm{~mm}$ diameter and $10 \mathrm{~mm}$ height). After keeping the samples in $100 \%$ relative humidity for $8 \mathrm{~h}$ to ensure cohesion, they were immersed in deionized water at $37^{\circ} \mathrm{C}$ for 10 days, to allow for the hydrolysis reaction of $\alpha$-TCP to CDHA to take place, according to the following reaction (Eq.1):

$$
3 \mathrm{Ca}_{3}\left(\mathrm{PO}_{4}\right)_{2}+\mathrm{H}_{2} \mathrm{O} \rightarrow \mathrm{Ca}_{9}\left(\mathrm{PO}_{4}\right)_{5}\left(\mathrm{HPO}_{4}\right) \mathrm{OH} \quad \text { (Eq. 1) }
$$

To obtain the biphasic hydroxyapatite/ $\beta$-TCP foams (BCP-Foam), instead of immersing the foams in water at $37^{\circ} \mathrm{C}$, they were unmoulded, immersed in a 1 wt $\%$ sodium bicarbonate solution $\left(\mathrm{NaHCO}_{3}\right.$, Sigma-Aldrich, St. Louis, USA) and autoclaved at $120^{\circ} \mathrm{C}$ and 1 atm for $30 \mathrm{~min}$. Subsequently, they were sintered at $1100^{\circ} \mathrm{C}$ for $9 \mathrm{~h}$ to obtain a BCP with a $80: 20 \mathrm{HA}: \beta-\mathrm{TCP}$ ratio. Additional information on this protocol is provided in the Supplementary Data (Fig. S1). The $\beta$-TCP foams ( $\beta$-TCP-Foam) were obtained by sintering the CDHA foams at $1100^{\circ} \mathrm{C}$ for $9 \mathrm{~h}$. 
For the in vitro studies, $5 \mathrm{~mm}$ diameter $\times 0.3 \mathrm{~mm}$ height discs of CDHA (CDHA-disc), $\beta$-TCP ( $\beta$ TCP-disc) and BCP (BCP-disc) were prepared, following the procedures described above for the foamed scaffolds, except for the foaming step, which was substituted by a mortar mixing for $1 \mathrm{~min}$.

\subsubsection{Preparation of robocast scaffolds}

To prepare the CDHA self-setting ink, a 30 wt\% aqueous solution of poloxamer 407 (P2443 Pluronic $^{\circledR}$ F-127, Sigma-Aldrich, St. Louis, USA) was mixed with the $\alpha$-TCP powder at a liquid to powder ratio of $0.65 \mathrm{~g} / \mathrm{g}$. A cylindrical CAD model of the scaffolds $(5 \mathrm{~mm}$ diameter and $10 \mathrm{~mm}$ height) was designed (Solidworks 2014, Dassault Systèmes SolidWorks Corp., Waltham, USA) and converted to a .STL 3D mesh, with a rectilinear pattern and an infill of 0.45 . Two different nozzles were used, with an inner diameter of 450 or $250 \mu \mathrm{m}$ for the CDHA-Rob-450 and CDHA-Rob-250 respectively. To print the scaffolds, the self-setting ink was introduced immediately after mixing into the cartridge of the robocasting device (Pastecaster, BCN3D Technologies, Barcelona, Spain). The scaffolds were left overnight in an incubator in $100 \%$ relative humidity at $37^{\circ} \mathrm{C}$, and subsequently immersed in deionized water at $37^{\circ} \mathrm{C}$ for a 10 days hardening period.

\subsubsection{Materials characterization}

The scaffolds were characterized in terms of phase composition, microstructure, specific surface area and porosity. Phase characterization was performed by X-ray diffraction using a diffractometer (D8 Advance, Bruker Corp., Billerica, USA) equipped with a Cu Ka anode operated at $40 \mathrm{kV}$ and 40 $\mathrm{mA}$. Data were collected in $0.02^{\circ}$ steps over the $2 \theta$ range of $10^{\circ}-80^{\circ}$ with a counting time of $2 \mathrm{~s}$ per step. Phase identification was accomplished comparing the experimental patterns to those of hydroxyapatite (JCPDS 09-0432), $\alpha$-TCP (JCPDS 09-0348) and $\beta$-TCP (JCPDS 09-0169). Quantitative phase composition analyses were carried out using DIFFRAC.EVA software, (Bruker Corp., Billerica, USA). The morphology of the scaffolds was assessed by micro-computed tomography (micro-CT, SkyScan 1172, Bruker microCT, Kontich, Belgium) at a voltage of $90 \mathrm{kV}$ and current of $112 \mu \mathrm{A}$, with a $\mathrm{Cu}-\mathrm{Al}$ filter. Images were acquired using an isotropic pixel size of 5 $\mu \mathrm{m}$. Reconstruction of cross-sections was done using software package NRecon (Bruker microCT, 
Kontich, Belgium). Calculations of macroporosity were performed with CTAn (Bruker microCT, Kontich, Belgium). The microstructure was characterized by scanning electron microscopy (Zeiss Neon40 EsBCrossBeam, Zeiss, Oberkochen, Germany). Prior to imaging, samples were coated with carbon to enhance conductivity. The SSA was determined by nitrogen adsorption using the BET (Brunauer-Emmett-Teller) method (ASAP 2020, Micrometrics Instrument Corp., Norcross, USA). Porosity and pore entrance size distribution were measured by mercury intrusion porosimetry (MIP, AutoPore IV, Micrometrics Instrument Corp., Norcross, USA). All samples were dried at $100^{\circ} \mathrm{C}$ for $2 \mathrm{~h}$ prior to measurement.

\subsection{In vivo study}

\subsubsection{Animal model and intramuscular implantation}

The capacity of the scaffolds to form ectopic bone was evaluated in a standardized intramuscular implantation model in canine. 12 adult beagle dogs (body weight 14-17 kg) were purchased from a professional stock breeder (Isoquimen S.L., Barcelona, Spain). All animal procedures were in compliance with the Guide for Care and Use of Laboratory Animals ${ }^{19}$ and the European Community Guidelines (Directive 2010/63/EU) for the protection of animals used for scientific purposes ${ }^{20}$. Ethical approval for the procedures was obtained from the local ethic committee (CEAAH 2338). On reception, animals were randomly divided into 2 groups of 6 dogs each, corresponding to two different sacrificial times ( 6 weeks/12 weeks). Afterwards, the animals were acclimatized to their local environment for 2 weeks prior to surgery.

For the surgical procedure, dogs were preanesthetized using an intramuscular injection of medetomidine and methadone. Anesthesia was induced by intravenous injection of propofol and diazepam and maintained with inhaled isoflorane in an oxygen carrier. During surgery the animals received an intravenous saline isotonic solution and intravenous injection of cefazolin. For the intramuscular implantation animals were placed in sternal recumbency and the lumbar areas were shaved and scrubbed with chlorhexidine gluconate solution for an aseptic preparation of the surgical field. Subsequently, one skin incision was performed on the lumbar region and fascia incisions were created in the paraspinal muscles bilaterally by scalpel. Using blunt dissection 
intramuscular pockets were created with a cranio-caudal direction and $4 \mathrm{~cm}$ lateral to the spinal axis. Each intramuscular pocket was filled with one of the above-mentioned cylindrical scaffolds (ø $5 \mathrm{~mm} \times 10 \mathrm{~mm}$ of height): CDHA-Foam, CDHA-Rob-450, CDHA-Rob 250, BCP-Foam, B-TCPFoam, applying a rotatory allocation system and a block design. Therefore, one construct of each material was implanted in each dog resulting in 5 scaffolds implanted per animal, leaving a distance of $5 \mathrm{~cm}$ between implants. All scaffolds had been previously sterilized by Gamma irradiation at a dose of $25 \mathrm{kG}$. Table 1 shows the list of implanted materials. Finally, muscular fasciae were closed with monofilament synthetic non-resorbable sutures for identification at harvest and subcutaneous tissue and skin incisions were closed layer by layer with monofilament synthetic absorbable sutures. Immediately after surgery, the animals received subcutaneously a prophylactic long-acting antibiotic (cefovecin) and pain relief (methadone and meloxicam).

Table 1. Summary of implanted samples

\begin{tabular}{|c|c|c|c|c|}
\hline \multirow[b]{2}{*}{ Architecture } & \multirow[b]{2}{*}{ Composition } & \multirow[b]{2}{*}{ Codes } & \multicolumn{2}{|c|}{ Intramuscular implantation } \\
\hline & & & 6 weeks & 12 weeks \\
\hline \multirow{3}{*}{ Foams } & Calcium deficient hydroxyapatite & CDHA-Foam & 6 & 6 \\
\hline & 80:20 hydroxyapatite: $\beta-T C P$ & BCP-Foam & 6 & 6 \\
\hline & $\beta$-TCP & $\beta$-TCP-Foam & 6 & 6 \\
\hline \multirow{2}{*}{ Robocast } & Calcium deficient hydroxyapatite & CDHA-Rob-450 & 6 & 6 \\
\hline & Calcium deficient hydroxyapatite & CDHA-Rob-250 & 6 & 6 \\
\hline
\end{tabular}

During the postoperative period, a non-steroidal anti-inflammatory drug was given subcutaneously to the animals for 7 days to prevent pain and inflammation. The animals were euthanized at 6 and 12 weeks post-implantation, by an intravenous injection of an overdose of pentobarbital sodium. A pre-euthanasia sedation of medetomidine was used for animal welfare reasons.

\subsubsection{Sample harvest and histological processing}

Immediately after euthanasia, the samples were harvested with their surrounding muscular tissue and fixed in $4 \%$ neutral buffered formalin solution for 72 hours. After fixation, they were dehydrated in an increasing series of ethanol solutions and embedded in four different graded mixtures of ethanol and methylmethacrylate resin (Technovit 7200, HeraeusKulzer GmbH, Hanau, Germany) 
under vacuum conditions. The specimens were subsequently photopolymerized with $2 \mathrm{~h}$ of white light and $4 \mathrm{~h}$ of ultraviolet light, obtaining blocks that were scanned by X-ray micro-CT. After microCT scanning, each block was divided transversally, perpendicular to the long axis of the implant, into two equal pieces. One piece was sectioned and polished (EXAKT Cutting \& Grinding System, EXAKT Advanced Technologies $\mathrm{GmbH}$, Norderstedt, Germany) prior to sputtering of the surface with carbon to enhance conductivity for backscattered scanning electron microscopy analysis (BSSEM). The other piece was sliced into $500 \mu \mathrm{m}$ thick sections, followed by a subsequent thinning to $50 \mu \mathrm{m}$ by grinding (Cutting \& Grinding System, EXAKT Advanced Technologies GmbH, Norderstedt, Germany). The sections were then stained with Goldner-Masson trichrome and Toluidine blue for histological observation under light microscopy.

\subsubsection{Histology and histomorphometry}

The micro-CT analysis (SkyScan 1172, Bruker microCT, Kontich, Belgium) of the tissue samples was performed using the same settings as for the scaffolds scanning described above. The 3D quantification of new bone formation and scaffold degradation was performed following a previously established protocol $^{21}$, where the BS-SEM images were used as a reference to determine the grey scale intensity thresholds to differentiate bone and calcium phosphate biomaterial in the micro-CT images. The following parameters were quantified:

a) Percentage of newly formed bone within the available macropore space: $\%$ newly formed bone $=($ bone volume $/$ available macropore volume $) * 100$

b) Percentage of scaffold degradation, calculated by subtracting the remaining scaffold volume from the initial scaffold volume prior to implantation:

$\%$ scaffold degradation $=[($ initial scaffold volume - final scaffold volume $) /$ initial scaffold volume $)]$ * 100

Moreover, the radius of each implanted scaffold was divided into five equal segments to define five concentric volumes of interest (VOIs). The percentage of newly formed bone was calculated in each VOI, to assess the distribution of the new ectopic bone formation.

The BS-SEM observations (Zeiss Neon40 EsBCrossBeam, Zeiss, Oberkochen, Germany) were made at $20 \mathrm{kV}$ to assess the presence, localization and maturity of the newly formed bone tissue and scaffold degradation based on its morphology and the different contrast levels. The number of 
samples that showed any bone formation per the total number of samples implanted (bone incidence rate) was recorded.

Stained histological sections were scanned using a light microscope (Nikon Eclipse E800, Nikon Corp.,Tokyo, Japan) fitted with a digital camera (ProgRes, Jenoptik AG, Jena, Germany) for a qualitative evaluation focused on fibrous tissue infiltration, presence of bone cells (osteoblasts, osteocytes and osteoclasts), grade of neovascularization and grade of peri-implant inflammatory reaction. Digital images were captured using an image analysis software (ProgRes CapturePro, Jenoptik AG, Jena, Germany).

\subsection{In vitro study}

\subsubsection{Cell culture}

To better understand the mechanisms leading to osteoinduction, rat mesenchymal stem cells (rMSCs) were cultured in direct contact with the different materials used in the in vivo study. rMSCs were isolated from tibias and femurs of Lewis rats and characterized by flow cytometry as described elsewhere ${ }^{22}$. Cells were expanded in Advanced Dulbecco's Modified Eagle Medium (AdvDMEM) supplemented with $10 \%$ fetal bovine serum (FBS), 20 mM4-(2Hydroxyethyl)piperazine-1-ethanesulfonic acid (HEPES) buffer, $2 \mathrm{mM} \mathrm{L-glutamine} \mathrm{(50} \mathrm{U/ml)} \mathrm{and}$ penicillin/streptomycin $(50 \mu \mathrm{g} / \mathrm{ml})$, all from Thermo Fischer Scientific Inc. (Waltham, USA). Cells at passages 3-4 were used in all experiments.

Discs were sterilized by immersion in $70 \%$ ethanol, and rinsed three times with Phosphate Buffered Saline (PBS, Gibco, Thermo Fisher Scientific Inc., Waltham, USA). Afterwards, samples were placed in 24-well plate and incubated with $2.5 \mathrm{ml}$ medium/well overnight.

\subsubsection{Cell differentiation: Real-time quantitative PCR}

Cells were seeded on the samples $\left(300\right.$ cells $\left./ \mathrm{mm}^{2}\right)$ and incubated for different time periods. The differentiation to osteoblastic phenotype was assessed by measuring gene expression of osteogenic markers (Table 2) by Real-Time quantitative Polymerase Chain Reaction (RT-qPCR). Total RNA was extracted at $6 \mathrm{~h}$ and 1 and 3 days using RNeasy Mini Kit (Qiagen GmbH, Hilden, 
Germany), as recommended by the manufacturer. For osteocalcin (OCN), a late osteogenic marker, 7 and 14 days were also monitored. Prior to RNA extraction, samples were transferred into new well plate and they were rinsed with PBS (Gibco, Thermo Fisher Scientific Inc., Waltham, USA). Total RNA was quantified by NanoDrop ND-1000 spectrophotometer (NanoDrop Products, Thermo Fisher Scientific Inc., Waltham, USA) and $120 \mu \mathrm{g}$ were used for synthesis of complementary DNA (cDNA) using the QuantiTect Reverse Transcription Kit (Qiagen GmbH, Hilden, Germany). cDNA templates were amplified using specific primers (Table 2) in SYBR Green RT-qPCR analyses using the QuantiTect SYBR Green RT-PCR Kit (Qiagen GmbH, Hilden, Germany) in an RT-PCR StepOnePlus (Applied Biosystems, Thermo Fisher Scientific Inc., Waltham, USA). The specificity of primers was ensured by melt curves analysis in all RT-qPCR runs. To verify the absence of contamination and genomic DNA, No-RNA control and No-RT enzyme control were evaluated in parallel. The expression of studied genes were normalised by expression of $\beta$-actin (housekeeping gene) and relative fold changes (FC) were related to tissue culture polystyrene (TCPS) at $6 \mathrm{~h}$ of culture. The following formula were used: $\mathrm{FC}=\mathrm{E}_{\mathrm{target}}{ }_{\mathrm{Cq}}$ target (TCPS $6 \mathrm{~h}$ - sample) $/ \mathrm{E}_{\text {housekeeping }}{ }^{\Delta \mathrm{Cq}}$ housekeeping (TCPS $6 \mathrm{~h}$ - sample), where $\mathrm{C}_{\mathrm{q}}$ is the median value of the quantification cycle of the triplicate of each sample and $E$ corresponds to the efficiency of amplification and is determined from the slope of the log-linear portion of the calibration curve, as $E=10^{(-1 / \text { slope })}$. The experiment was performed in two independent runs.

Table 2. Primers' sequences used for RT-qPCR

\begin{tabular}{cccc}
\hline Gene & $\begin{array}{c}\text { Gene } \\
\text { symbol }\end{array}$ & $\begin{array}{c}\text { Forward primer sequence } \\
\left(5^{\prime} \text { to } 3^{\prime}\right)\end{array}$ & $\begin{array}{c}\text { Reverse primer sequence } \\
\left(5^{\prime} \text { to } 3^{\prime}\right)\end{array}$ \\
\hline B-actin & ACTB & CCCGCGAGTACAACCTTCT & CGTCATCCATGGCGACT \\
Bone morphogenetic protein-2 & BMP-2 & CCCCTATATGCTCGACCTGT & AAAGTTCCTCGATGGCTTCTT \\
Alkaline phosphatase & ALP & GCACAACATCAAGGACATCG & TCAGTTCTGTTCTTGGGGTACAT \\
Collagen I & Coll & CATGTTCAGCTTTGTGGACCT & GCAGCTGACTTCAGGGATGT \\
Osteonectin & ONN & GTTTGAAGAAGGTGCAGAGGA & GGTTCTGGCAGGGGTTTT \\
Osteopontin & OPN & CGGTGAAAGTGGCTGAGTTT & GGCTACAGCATCTGAGTGTTTG \\
Osteocalcin & OCN & ATAGACTCCGGCGCTACCTC & CCAGGGGTCTGGGTAGG \\
\hline
\end{tabular}




\subsection{Statistical analysis}

The histomorphometric and in vitro results are presented as mean values \pm standard error. Statistical comparisons between experimental groups were performed using One-way repeated measures ANOVA followed by Tukey's post hoc test in GraphPad Prism software (GraphPad Software Inc., La Jolla, USA). A pair-wise comparison result of $p<0.05$ was considered statistically significant. 


\section{Results}

\subsection{Materials characterization}

The textural properties of the different scaffolds including total porosity and macroporosity, determined by MIP and micro-CT, as well as, the BET specific surface area are summarized in Table 3. The architecture of the foamed and 3D-printed scaffolds, together with the microstructure of the different materials can be seen in the SEM images in Fig.1A and the pore size distribution, measured by MIP, is shown in Fig. 1B. Similar microstructures were observed for the CDHA-Foam, CDHA-Rob-450 and CDHA-Rob-250 with the typical entangled network of needle-like CDHA nanocrystals (Fig. 1A), which resulted in high SSA, around $35 \mathrm{~g} / \mathrm{m}^{2}$ (Table 3). As a consequence, the three CDHA groups presented nanosized pores (Fig. 1B). However, whereas the robocast samples displayed a nanopore size distribution ranging from 0.006 to $0.1 \mu \mathrm{m}$, the CDHA-Foam group showed a wider and continuous distribution, covering both the nano and the microscale. In contrast, the two sintered ceramics (BCP-Foam and $\beta$-TCP-Foam) consisted of polyhedral crystals with smooth faces (Fig. 1A), displaying low SSA values (Table 3), an absence of nanoporosity and micropores centered around $1 \mu \mathrm{m}$ for the $\beta$-TCP-Foam group and at $1.2 \mu \mathrm{m}$ for the BCP-Foam group.

All scaffolds presented similar amounts of macroposity and total porosity as shown in Table 3, although different pore sizes and, more markedly, different pore entrance sizes (Fig. 1, Table 3), larger for the robocast scaffolds. Moreover, as observed in Fig. 1A, the foamed scaffolds presented a very similar architecture with interconnected spherical macropores, irrespective of its biomimetic or sintered nature.

Table 3. Porosity and SSA of the different scaffolds

\begin{tabular}{ccccccc}
\hline \multirow{2}{*}{ Materials } & \multicolumn{7}{c}{ Porosity } & $\begin{array}{c}\text { \% Total } \\
\text { Porosity by } \\
\text { MIP }\end{array}$ & $\begin{array}{c}\text { \% Macroporosity } \\
\text { by MIP }(>\mathbf{1 0} \boldsymbol{\mu m})\end{array}$ & $\begin{array}{c}\text { \% Macroporosity } \\
\text { by micro-CT }\end{array}$ & $\begin{array}{c}\text { Macropore } \\
\text { entrance size } \\
\text { by MIP }(\boldsymbol{\mu m})^{*}\end{array}$ & $\begin{array}{c}\text { Macropore size } \\
\text { by micro-CT } \\
(\boldsymbol{\mu m})^{*}\end{array}$ & $\begin{array}{c}\text { SSA } \\
\left(\mathbf{m}^{2} / \mathbf{g}\right)\end{array}$ \\
\hline CDHA-Foam & 76.5 & 49.5 & 52.3 & 70 & 227.0 & 38.49 \\
CDHA-Rob-450 & 64.8 & 46.5 & 47.4 & 350 & 409.7 & 32.02 \\
CDHA-Rob-250 & 65.4 & 48.7 & 54.1 & 200 & 288.7 & 32.35 \\
BCP-Foam & 71.8 & 47.2 & 47.4 & 50 & 214.8 & 0.42 \\
B-TCP-Foam & 64.6 & 43.2 & 48.9 & 40 & 232.0 & 0.46
\end{tabular}

*Whereas by MIP provided the average entrance size of the macropores, the value measured by micro-CT corresponds to the average pore size itself. 
A)

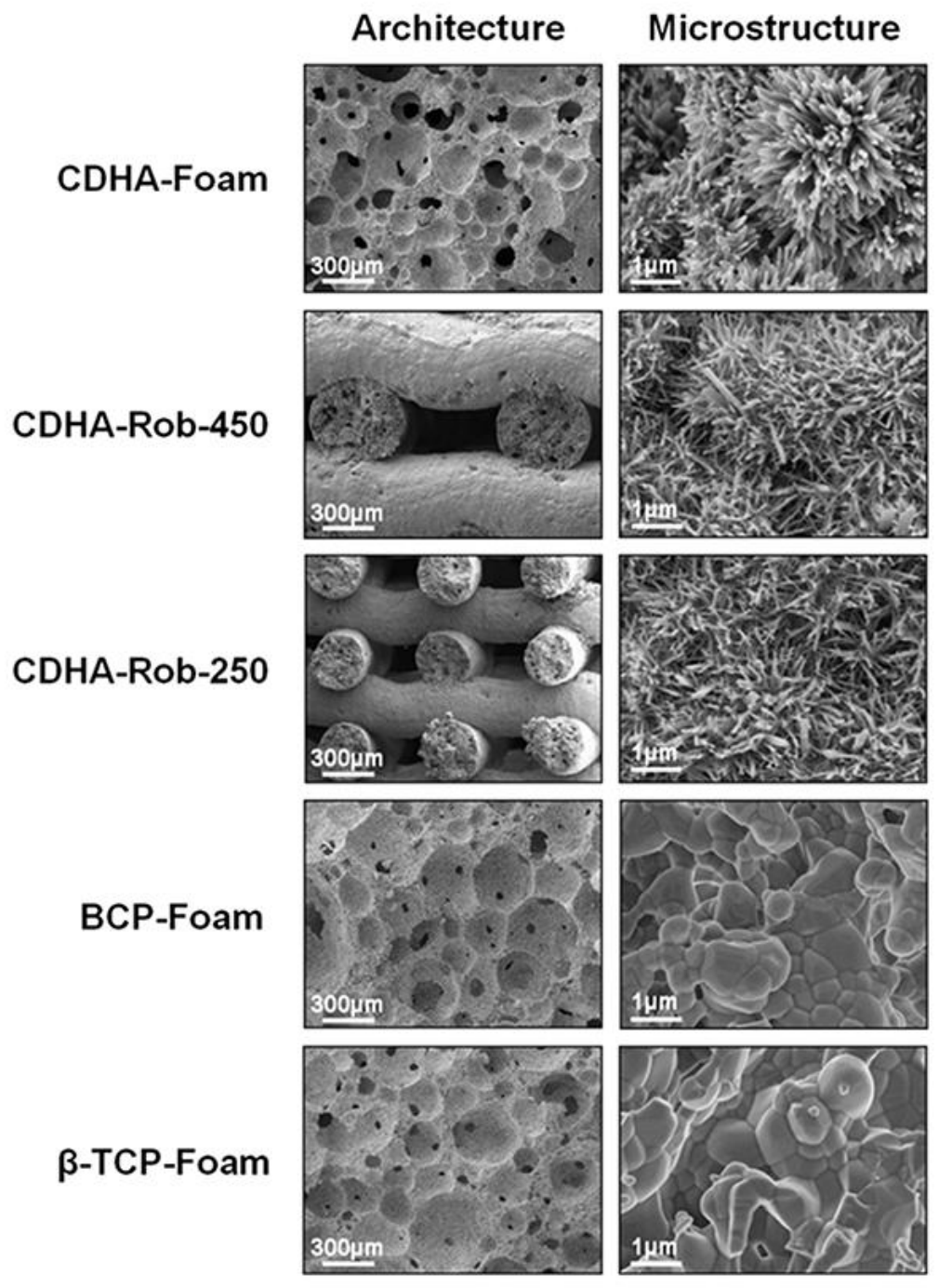

B)

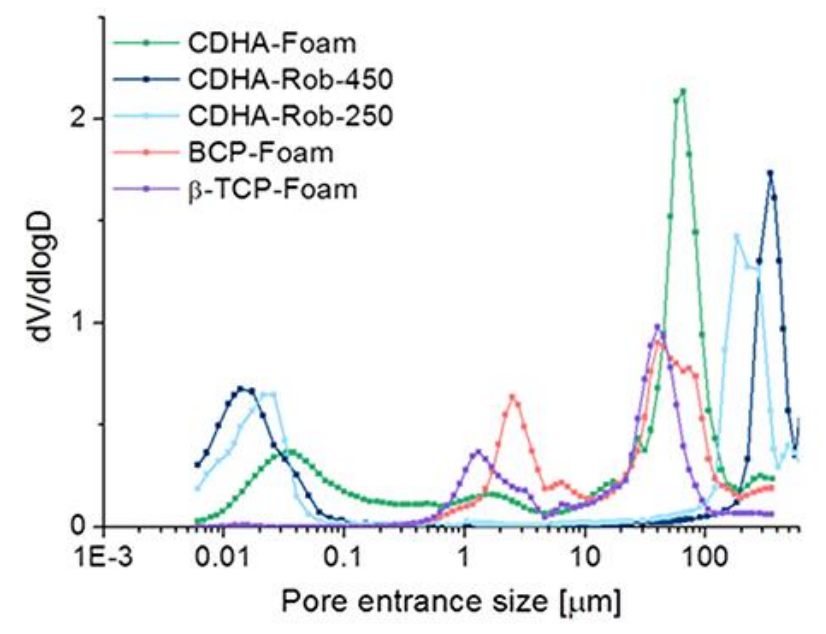

Figure 1. A) Scanning electron micrographs of the five implanted materials: CDHA-Foam, BCP-Foam and $\beta$-TCP-Foam groups showed similar architecture, consisting of open and interconnected spherical concave macropores, while the robocast scaffolds presented open and interconnected prismatic convex macropores. The microstructure of the CDHA scaffolds consisted of the typical network of entangled needle-like nanocrystals, while the sintered ceramics showed polyhedral smooth crystals. B) Pore entrance size distribution of the five implanted materials as determined by MIP. 
As shown by the XRD patterns (Supplementary Data, Fig. S2), the CDHA-Foam and the CDHARob scaffolds consisted of a poorly crystalline apatitic phase, as indicated by the broad peaks, typical of biomimetic apatites. Small amounts of unreacted $\alpha$-TCP were detected, and quantified to be $2 \%$ and $3 \%$ for CDHA-Foam and CDHA-Rob scaffolds, respectively. The sintered scaffolds exhibited sharper peaks typical of highly crystalline materials. $\beta$-TCP-Foam scaffolds consisted of phase pure $\beta$-TCP and BCP-Foam consisted of a mixture of $82.7 \% \mathrm{HA}$ and $17.3 \% \beta-\mathrm{TCP}$.

\subsection{Intramuscular implantation}

All surgeries were uneventful, all animals had a normal recovery after surgery and their postoperative course proceeded without any complications. No clinical evidence of inflammatory response or adverse tissue reaction around implants was observed during the experimental period or at retrieval. Histology showed low-grade peri-implant inflammatory reaction at both time points for all scaffolds, which were surrounded by well-vascularized muscle tissue. A small number of inflammatory cells, mainly limphoplasmocitary cells, were found and no fibrous capsule formation was observed, indicating the good biocompatibility of the different scaffolds. At 6 weeks fibrous tissue infiltration with abundant neovascularization was observed within the interconnected macropores, both in the foamed and the robocast scaffolds. Collagen fibres appeared to be oriented following the walls of the macropores (Fig. 2).
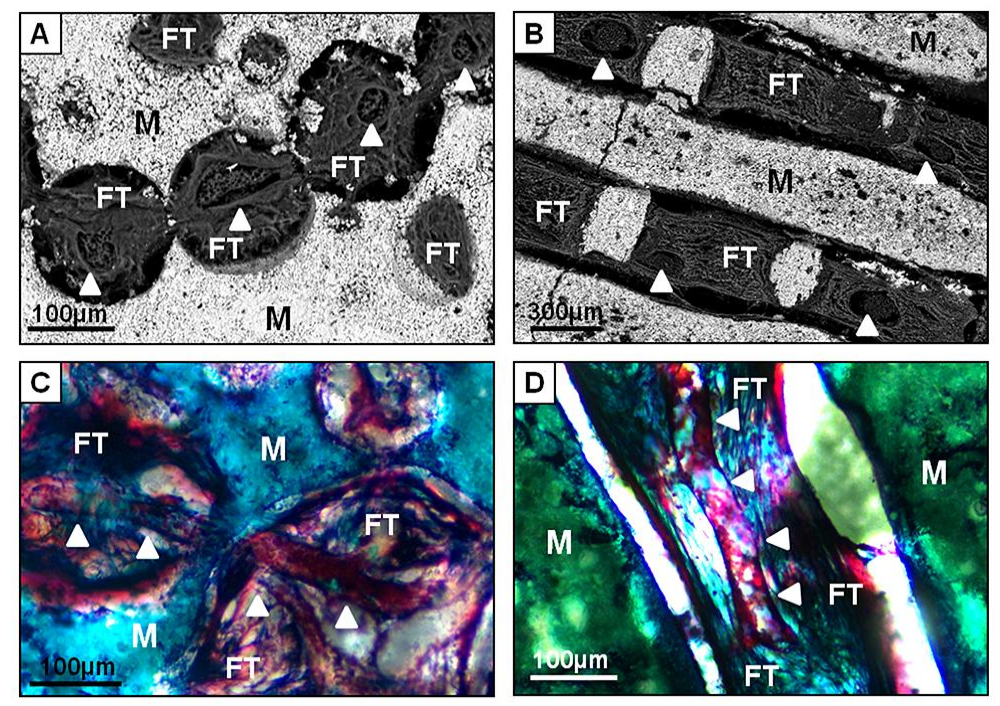

Figure 2. Backscattered scanning electron micrographs $(A, B)$ and micrographs of undecalcified Goldner-Masson trichrome stained sections $(C, D)$ of a representative foam scaffold $(A, C)$ and a robocast scaffold $(B, D)$ after 6 weeks of implantation. All foams and robocast materials showed fibrous tissue infiltration and blood vessels (arrow heads) within the interconnected macropores at 6 weeks. M=Material, FT=Fibrous Tissue. 
The results for new ectopic bone formation in the different scaffolds are summarized in Fig. 3. At 6 weeks new bone was detected only in the CDHA-Foam group, in 4 out of 6 animals, while no signs of bone formation were found neither in the sintered ceramic foams nor in the biomimetic robocast scaffolds. In this group, although no statistically significant differences between the five concentric VOls were noted, the ectopic bone was mainly found in the outer and middle regions (Supplementary Data, Fig. S3). No ectopic bone was observed on the outside surface of the cylinders. The newly formed bone was predominately located in the concavities of the macropores and in direct contact with the biomaterial, as shown in Fig. 4B, and consisted of a mineralized bone matrix, with osteocytes inside lacunae, and aligned osteoblasts laying down new osteoid (Fig.4D). The newly deposited bone was basically woven bone, with some areas of lamellar bone and a few Haversian structures (Fig. 4C). No endochondral ossification was detected during the ectopic bone formation process.

A) Bone incidence

\begin{tabular}{ccc}
\hline Materials & 6 weeks & 12 weeks \\
\hline CDHA-Foam & $4 / 6$ & $6 / 6$ \\
CDHA-Rob-450 & $0 / 6$ & $1 / 6$ \\
CDHA-Rob-250 & $0 / 6$ & $0 / 6$ \\
BCP-Foam & $0 / 6$ & $4 / 6$ \\
B-TCP-Foam & $0 / 6$ & $1 / 6$ \\
\hline
\end{tabular}

\section{B) Ectopic bone formation}

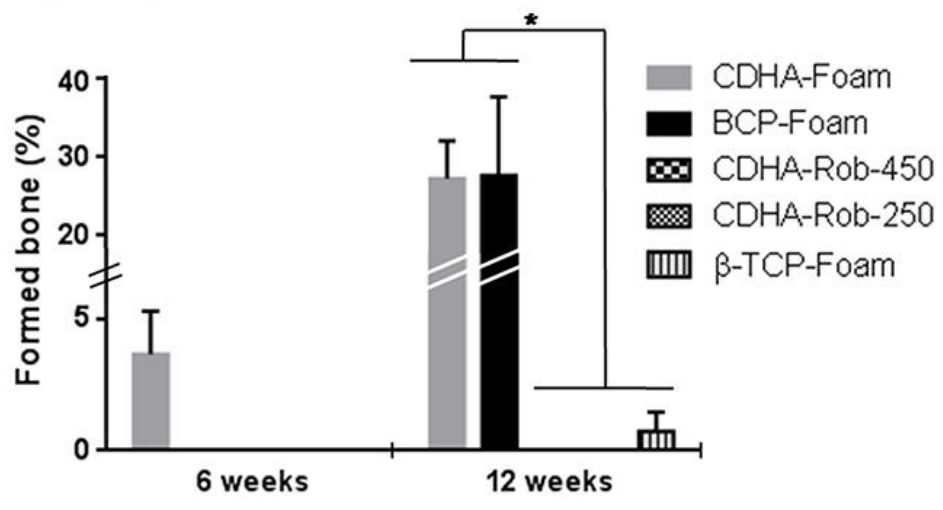

\section{C) Ectopic bone distribution at 12 weeks}
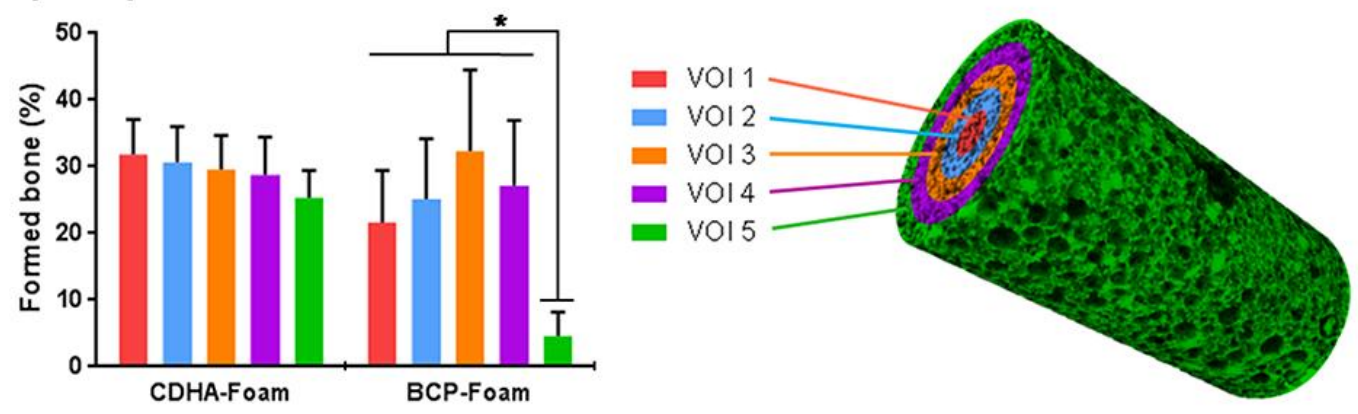

Figure 3. A) Bone incidence after 6 and 12 weeks of implantation. B) Histomorphometrical results: $\%$ of newly formed bone within the available macropores space at 6 and 12 weeks post-implantation, as measured by $\mu$-CT. The mean value for the CDHA-Rob-450 group was $0.01 \pm 0.01 \%$, hereby this is not appreciable in the graphic. $\left(^{*}\right)$ denotes groups with statistically significant differences $(p<0.05)$. C) Histomorphometrical results: $\%$ of newly formed bone within the available macropores space within the five volumes of interest (VOls) after 12 weeks of implantation in CDHA-Foam and BCPFoam scaffolds. The bone was uniformly distributed over the entire implant in CDHA-Foams, whereas in BCP-Foams there was very little bone in the outer VOI and it was concentrated mostly in the middle VOls. $\left(^{*}\right)$ denotes groups with statistically significant differences $(p<0.05)$. 

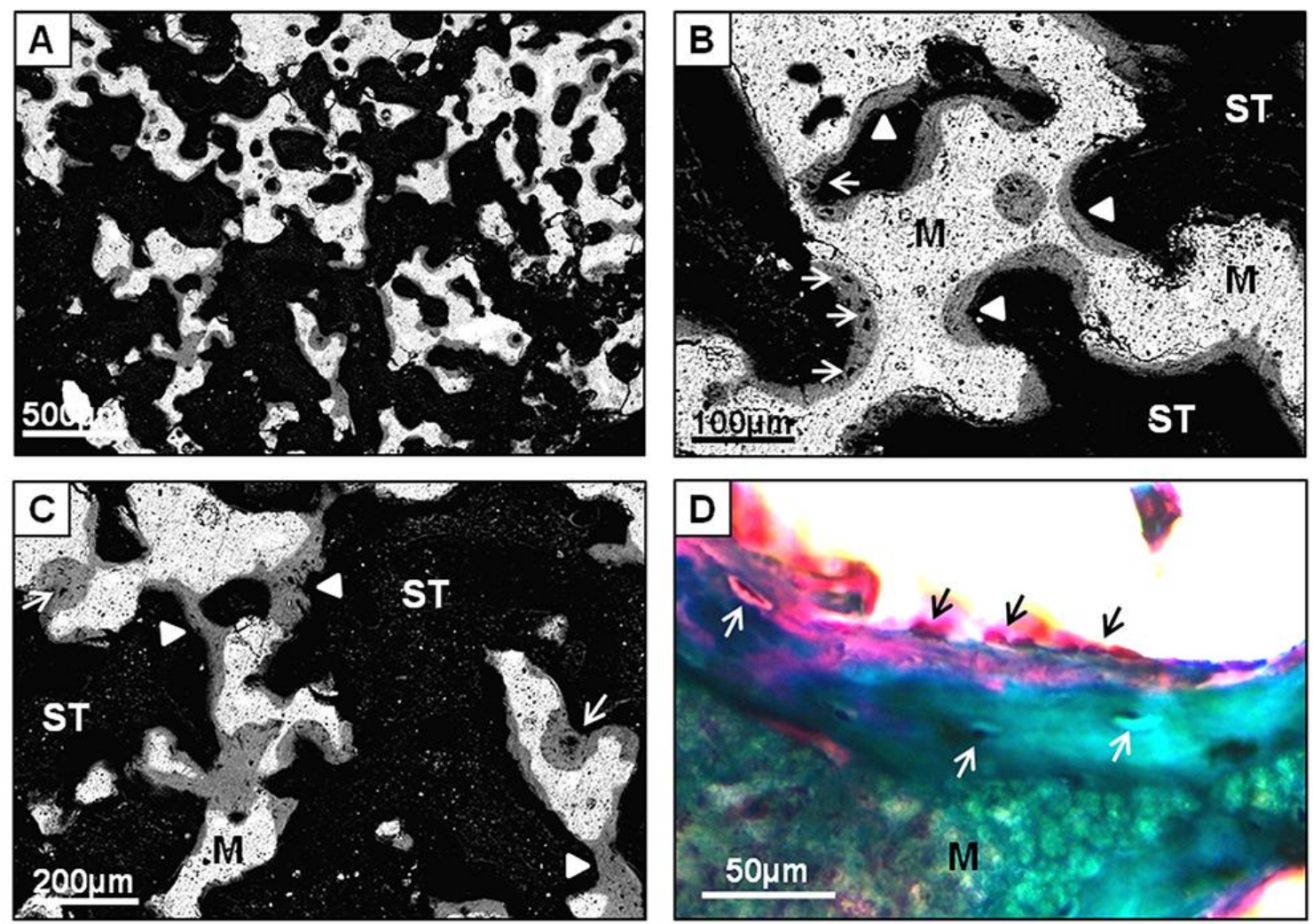

Figure 4. $(A, B, C)$ Backscattered scanning electron micrographs of undecalcified section of a CDHA-Foam scaffold after 6 weeks of implantation. B) The newly formed bone (white arrow heads) was arranged concentrically to the concave surface of scaffold macropores and always in direct contact with the material. Note the formation of well-mineralized bone matrix with some lacunae (white arrows). C) The new ectopic bone (white arrow heads) was basically woven bone with some osteon-like structures (white arrows). M=Material, ST=Soft tissue (in black). D) Micrograph of an undecalcified Goldner-Masson trichrome stained section of a CDHA-Foam scaffold after 6 weeks of implantation showing wellmineralized bone matrix (in green), with osteocytes inside lacunae (white arrows), and aligned osteoblasts (black arrows) laying down new osteoid (in pink). M=Material.

At 12 weeks, a significant amount of new ectopic bone was observed within the macropores of the CDHA-Foam group, in 6 out of 6 animals (27.98 $\pm 4.80 \%$ ectopic bone), as well as in the BCPFoam group, in 4 out of 6 animals (28.30 $\pm 10.11 \%$ ectopic bone) (Fig. 3B.). The bone was uniformly distributed over the entire implant in CDHA-Foams with no significant differences between the five concentric VOls at this time point (Fig. 3C and Fig. 5A). However, BCP-Foams exhibited a repetitive pattern, with almost no bone formation in the outer VOI (VOI 5), and most bone concentrated in the middle VOI (VOI 3) (Fig. 3C and Fig. 5B). Moreover, distinctive bone formation patterns were observed in these two groups, as shown in Fig. 5. Whereas in the BCPFoam group the new bone was deposited on top of the surface of the scaffold, filling the macropore space (Fig. 5B), in the CDHA-Foam group the bone seemed to replace the scaffold (Fig. 5A).This 
observation was confirmed when comparing the amount of pore volume before and after implantation in these two groups. Thus, even if both groups showed a similar amount of new ectopic bone formation, the fraction of total pore volume at 12 weeks decreased with $4.56 \%$ in the BCP-Foam group while it increased with $4.77 \%$ in the CDHA-Foam group when compared to the original scaffolds. The new ectopic bone was observed within macropores from 10 to $300 \mu \mathrm{m}$ in diameter in both groups and it consisted in mature lamellar bone with some areas of osteonal bone following a Haversian pattern (Fig. 5A/5B and Fig. 6A/6B/6D/6E). Moreover, abundant extracellular calcified bone matrix was observed within the micropores $(<10 \mu \mathrm{m})$ of these two groups as shown in Fig. 6G/6H.

New ectopic bone was found just in 1 animal out of 6 in the CDHA-Rob-450 and $\beta$-TCP-Foam groups, in a small amount in both cases (Fig. 3A/3B and Fig. 5C/5D). In CDHA-Rob-450 bone was mostly found in the corners, which is the only part of robocast samples with concavities (Fig. 5D and Fig. 6C/6F). CDHA-Rob-250 scaffolds did not show any ectopic bone formation at 12 weeks (Fig. 3A/4B and Supplementary Data, Fig.S4).

Some multinucleated osteoclast-like cells were consistently observed resorbing the materials in all scaffolds. However, a qualitative histological assessment identified a greater number of osteoclastlike cells in the CDHA-Foam and BCP-Foam scaffolds (Fig. 6I/6J, respectively) than in the CDHARob and $\beta$-TCP-Foam samples at both time points. 

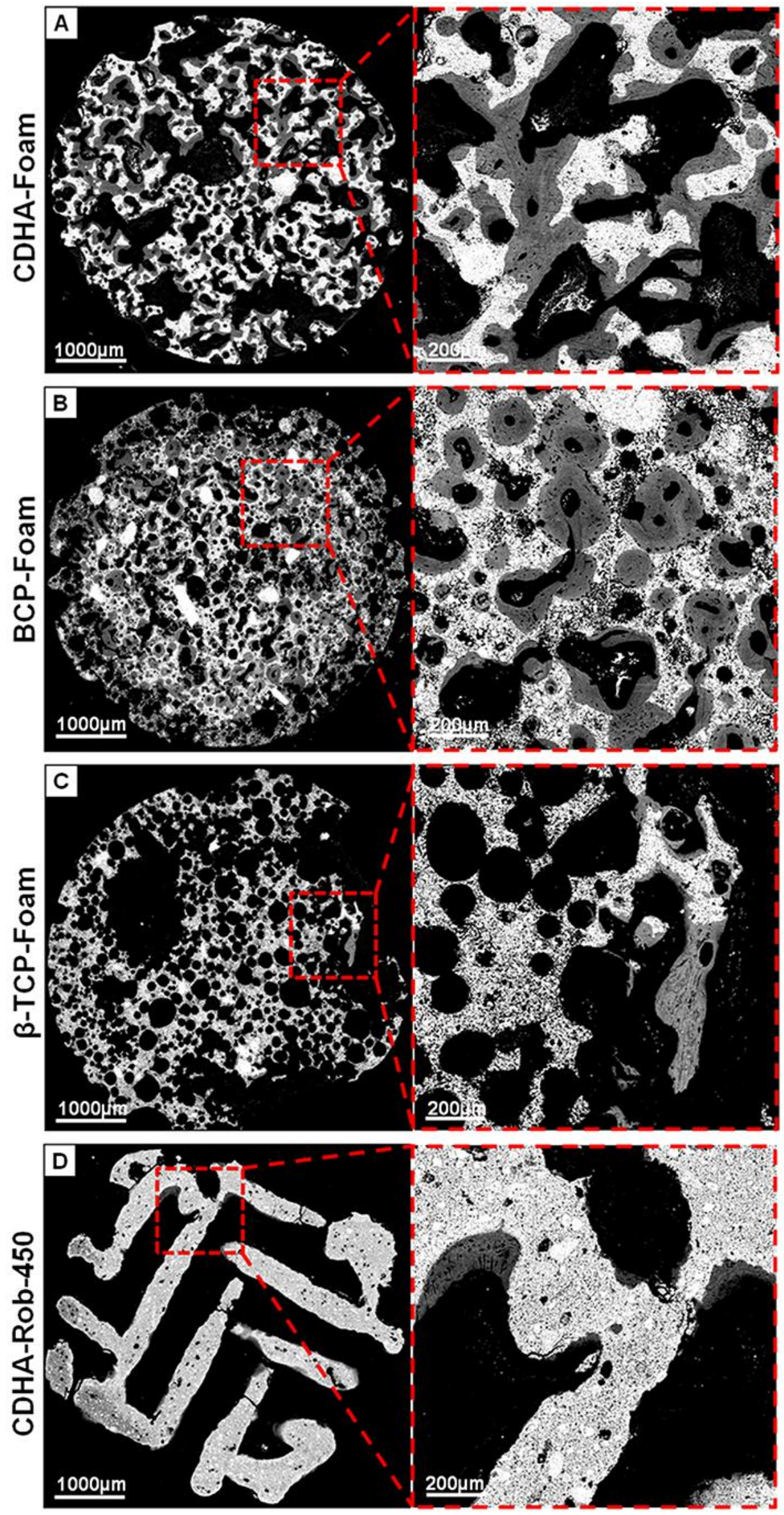

Figure 5. Backscattered scanning electron micrographs after 12 weeks of implantation of studied scaffolds: A) CDHA-Foam. B) BCP-Foam. C) $\beta$-TCP-Foam. D) CDHA-Rob-450 robocast scaffold. 

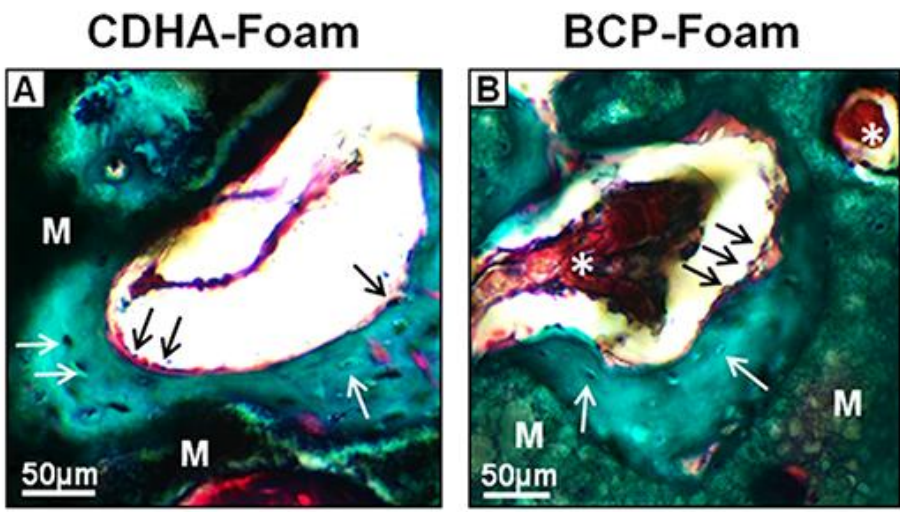

\section{CDHA-Rob-450}
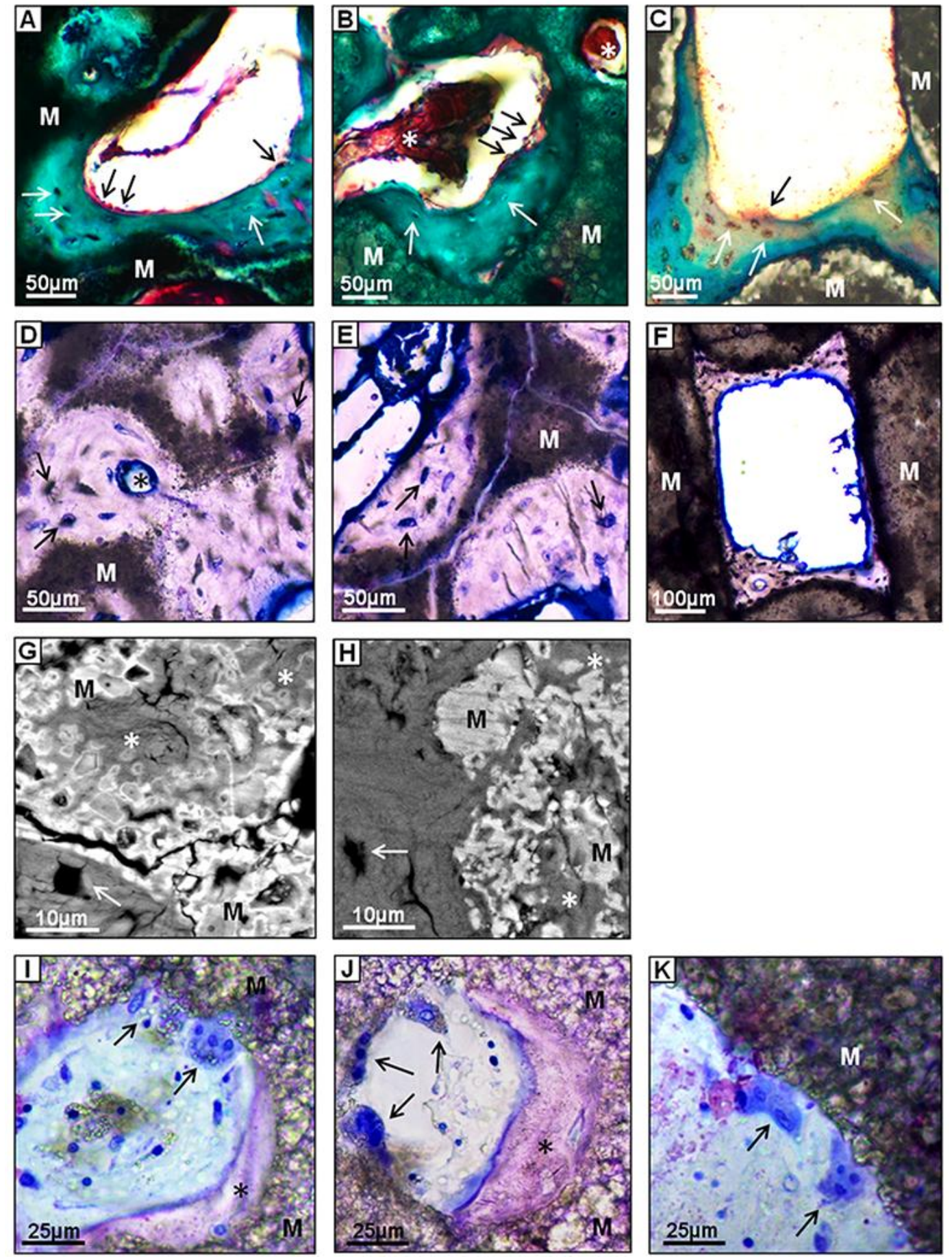

Figure 6. $(A, B, C)$ Micrographs of undecalcified Goldner-Masson trichrome stained sections of CDHA-Foam, BCP-Foam and CDHA-Rob-450 scaffolds after 12 weeks of implantation showing well-mineralized bone matrix (in green), with osteocytes inside lacunae (white arrows), and aligned osteoblasts (black arrows) laying down new osteoid (pink). $M=$ Material, Asterisk=blood vessel. (D,E,F) Micrographs of undecalcified Toludine blue stained sections of CDHA-Foam, BCP-Foam and CDHA-Rob-450 scaffolds after 12 weeks of implantation showing well-mineralized bone matrix (in purple), with osteocytes inside lacunae (black arrows) with bone canaliculi. In the CDHA-Rob-450, bone was mostly found in the corners, which is the only part of robocast samples with concave-like regions. $M=M a t e r i a l$, Asterisk=blood vessel. (G,H) Backscattered scanning electron micrographs of CDHA-Foam and BCP-Foam scaffolds after 12 weeks of implantation showing calcified bone matrix (asterisk) within micropores, next to an osteocyte (white arrow) located within a macropore. M=Material. $(\mathrm{I}, \mathrm{J}, \mathrm{K})$ Micrographs of undecalcified Toluidine blue stained sections of CDHA-Foam, BCPFoam and CDHA-Rob-450 scaffolds after 12 weeks of implantation showing multinucleated osteoclast-like cells (black arrows) resorbing the material with intracellular scaffold particles. M=Material, Asterisk=Bone matrix. 
The results concerning scaffold resorption of the different groups are summarized in Fig. 7A. A higher degradation was observed for the CDHA-Foam scaffolds compared to the other groups at 6 weeks, whereas at 12 weeks CDHA-Foam and $\beta$-TCP-Foam showed a similar degradation, significantly higher than that of CDHA-Rob-450 and 250, and the BCP-Foam groups (Fig. 7A). Noteworthy, the degradation of the CDHA-Foam scaffolds was progressive and homogeneous, while that showed by the $\beta$-TCP-Foam scaffolds was more heterogeneous, leaving big holes within the constructs, as revealed by the micro-CT images (Fig. 7B).

A)

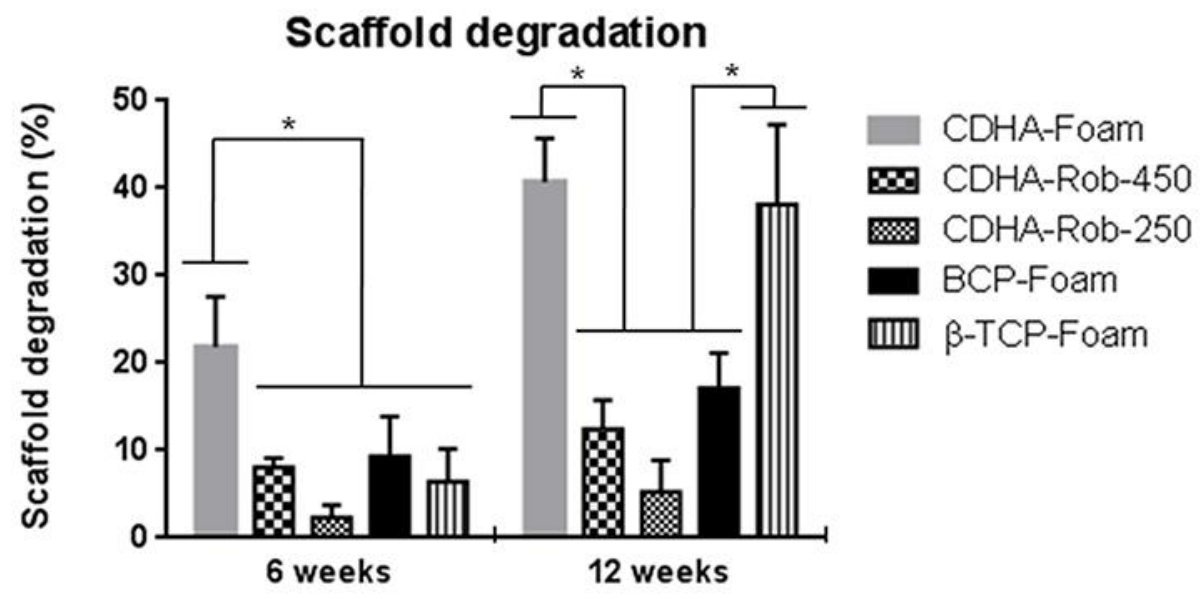

B)
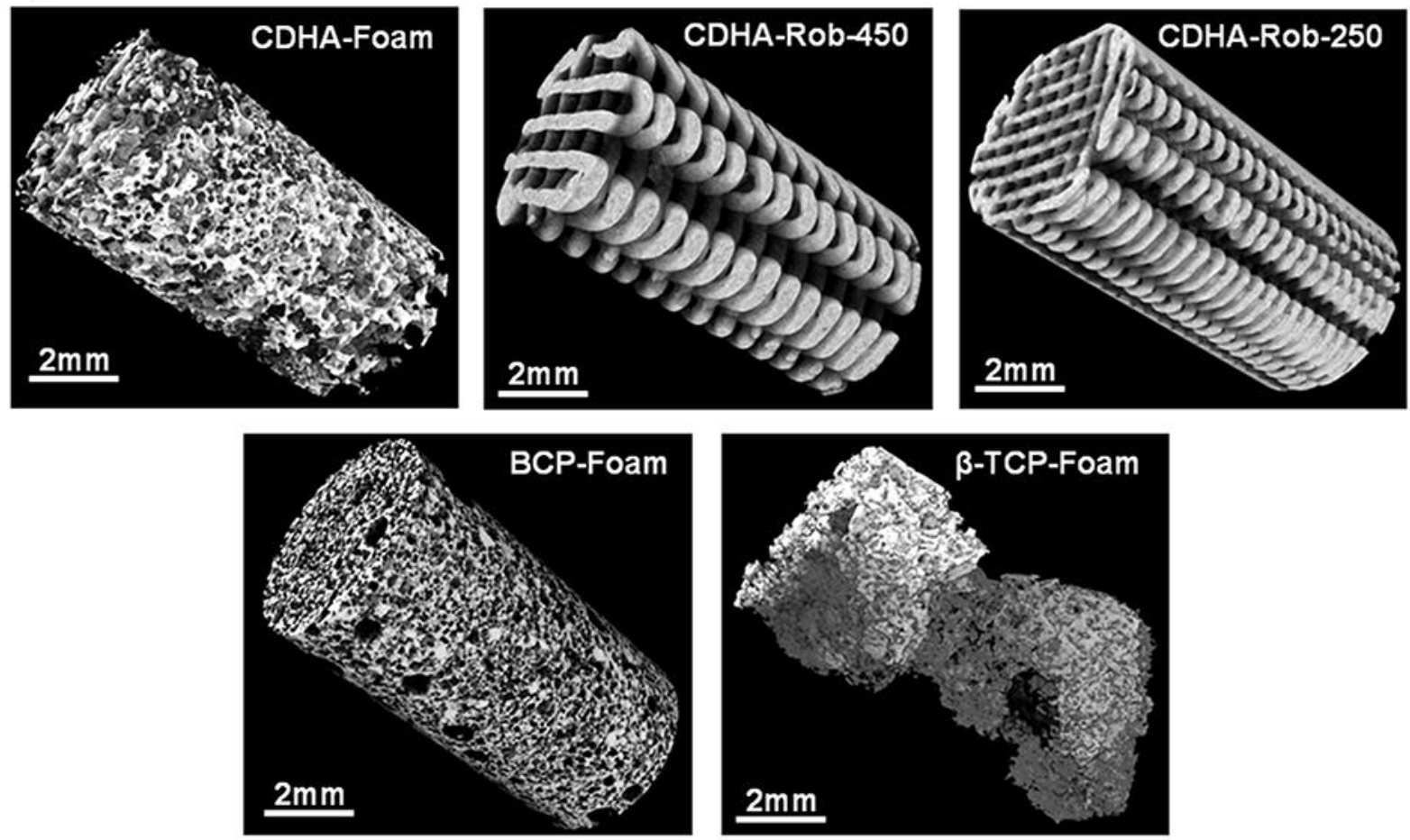

Figure 7. A) Histomorphometry results: \% of scaffold degradation after 6 and 12 weeks of implantation. $\left(^{*}\right)$ denotes groups with statistically significant differences $(p<0.05)$. B) Micro-CT 3D reconstructions of implanted scaffolds after 12 weeks of implantation. 


\subsection{In vitro study: cell differentiation (RT-qPCR)}

The expression profile of osteogenic markers of rMSCs was analysed in planar discs of the different materials used to fabricate the scaffolds, namely CDHA, BCP and $\beta$-TCP. The physicochemical properties of the discs are summarized in the Supplementary Data (Fig. S5). The results obtained by RT-qPCR analysis are displayed in Fig.8. The expression of BMP-2, OPN and OCN was significantly higher for cells cultured on CDHA compared to the sintered ceramics at all time-points, except for OCN at 6h. ALP, Col I and ONN expression followed similar trends, presenting the highest values at $6 \mathrm{~h}$ and decreasing over time. Specifically, the expression of ALP for cells cultured on CDHA was significantly higher than on sintered ceramics at $6 \mathrm{~h}$, while $\beta$-TCP showed significantly higher levels at 3 days compared to CDHA and BCP. Col I gene expression was significantly higher for cells cultured on CDHA compared to BCP at day 1. The expression of ONN by cells cultured on CDHA was significantly higher than on $\beta$-TCP at $6 \mathrm{~h}$ and significantly higher than on the two sintered ceramics at day 1. Finally, for $\mathrm{OCN}$, a late osteogenic marker, the analysis was prolonged until 7 and 14 days, finding a higher overexpression of OCN when cells were cultured on CDHA compared to the sintered ceramics at 7 days, whereas at 14 days similar values were found on CDHA and BCP, both higher than on $\beta$-TCP. 
BMP-2

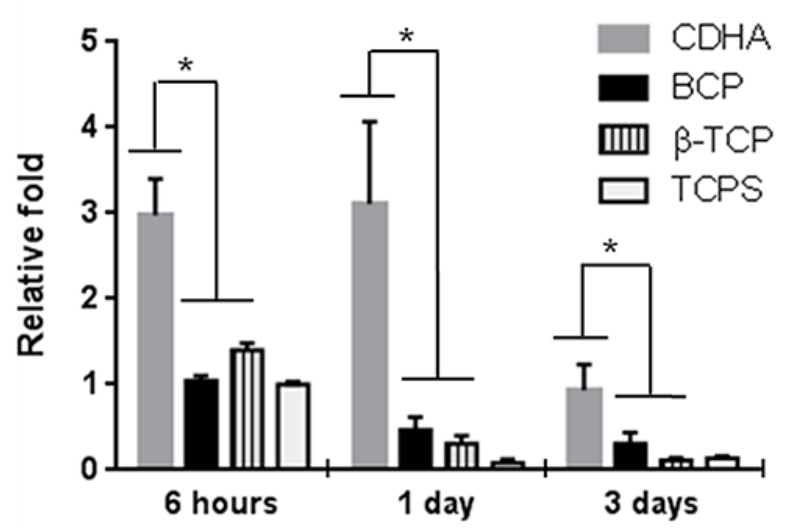

Col I
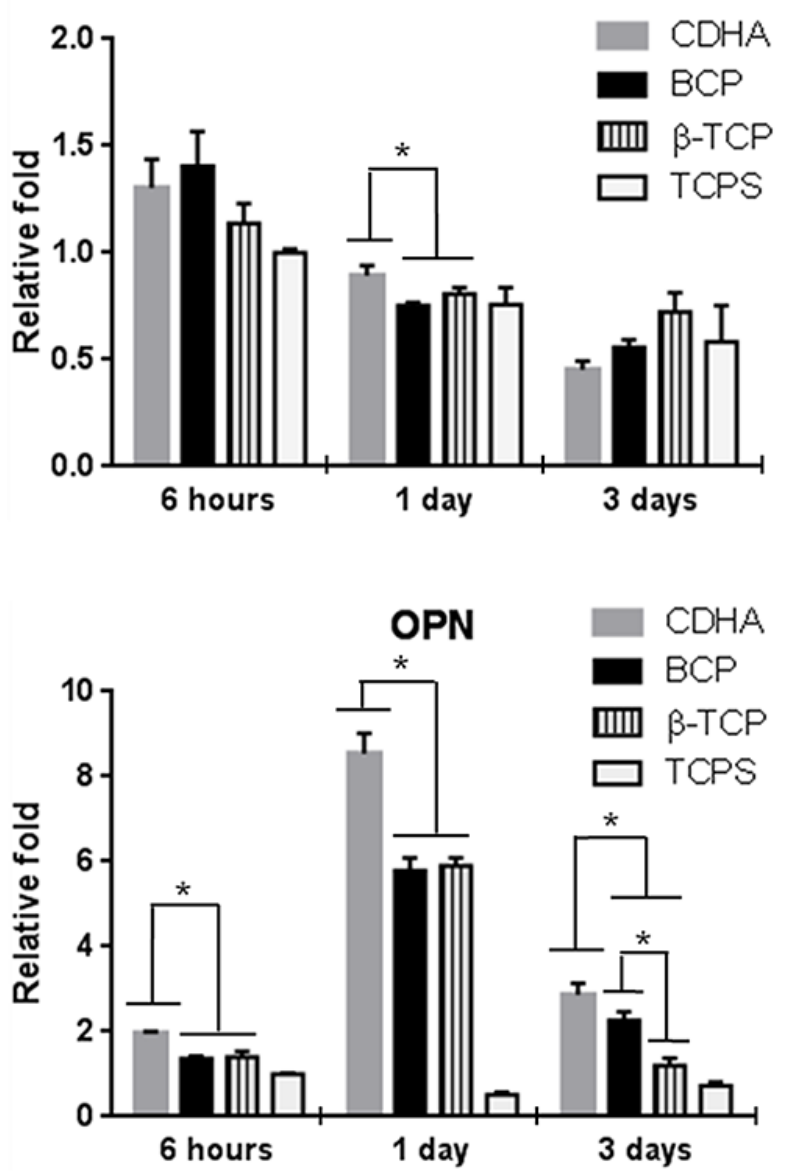

ALP

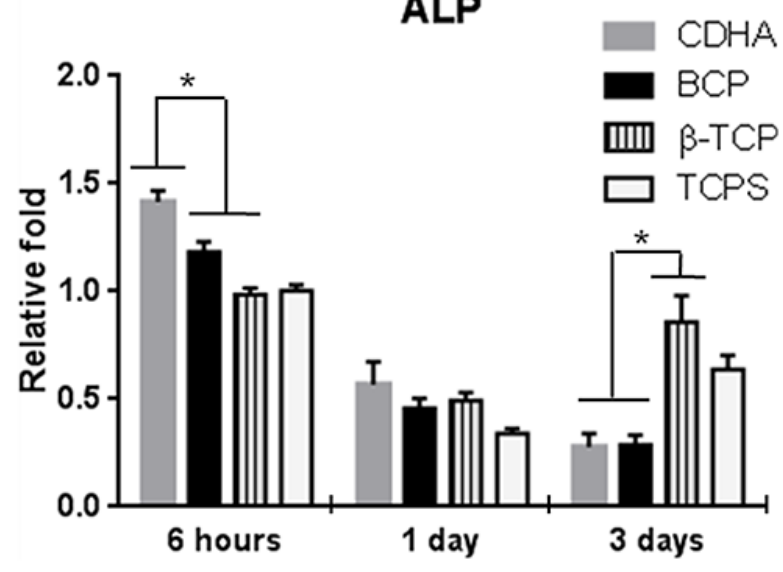

ONN

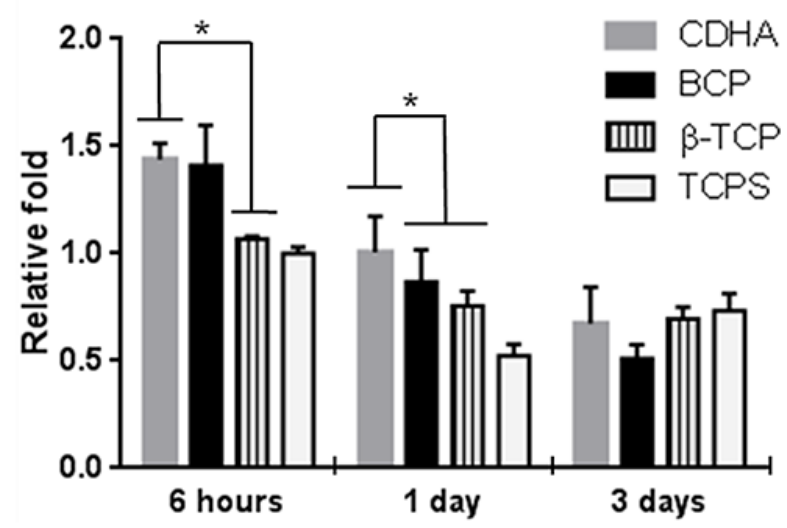

OCN

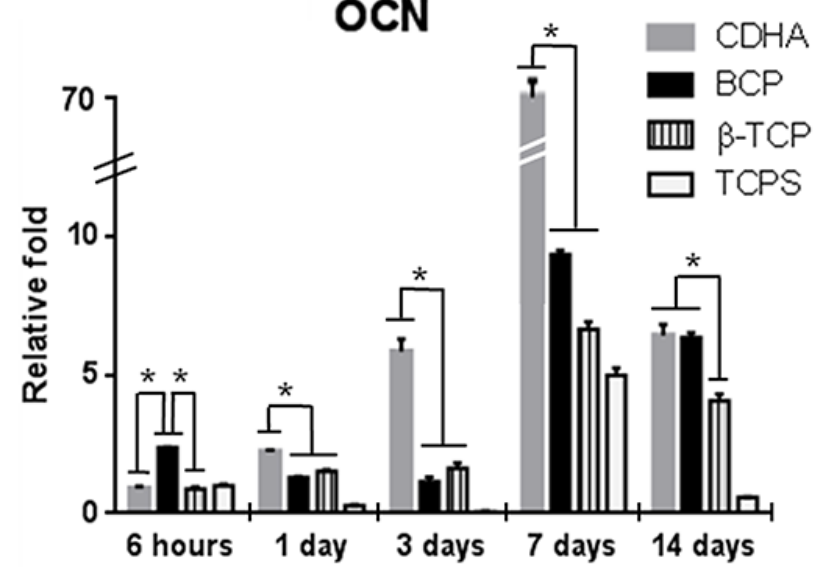

Figure 8. Gene expression of osteogenic markers of rMSCs cultured on planar discs of the different materials used to fabricate the scaffolds (CDHA, BCP and $\beta$-TCP) and measured by RT-qPCR. TCPS was used as a reference. Cells were cultured in basic medium. Statistical comparisons were performed between CaP materials. $\left(^{*}\right)$ denotes groups with statistically significant differences $(p<0.05)$. 


\section{Discussion}

The main goal of the present study was to obtain a better understanding of the role of textural properties and macropore architecture in osteoinduction, with the aim to shed light on the behaviour of biomimetic nanostructured materials compared to conventional sintered ceramics. Previous results pointed to microporosity and SSA as critical factors for the material-associated osteoinduction, although only a limited range of porosities and SSA had been analysed up to this moment. In this work, we have been able to extend this range to the nanoscale porosity, in combination with different macropore arquitectures. The results showed that both parameters are relevant, as foams induced ectopic bone formation to a much higher extent than robocast scaffolds, and amongst them, biomimetic nanostructured CDHA produced a higher incidence and accelerated bone formation when compared to microstructured sintered calcium phosphates. Moreover, different patterns of bone formation were observed, as will be discussed below.

\subsection{Animal model and surgical protocol}

The intrinsic osteoinductive potential of a biomaterial, e.g., its capacity to trigger the differentiation of mesenchymal stem cells to the osteoblastic lineage, can be proved by its ability to stimulate new bone formation when implanted ectopically in a host animal. In the present study the intramuscular implantation in a canine model was chosen, as ectopic bone formation is a species-dependent phenomenon, being more frequent in large (dog, sheep and goat) than in small animals (mouse, rat and rabbit) ${ }^{23-26}$. The higher vascularization of muscle compared to subcutaneous tissue was the reason for choosing this implantation site 24,26 .

Concerning the surgical protocol, it is worth mentioning that in our study a non-steroidal antiinflammatory drug (NSAIDs) was administered to the animals post-operatively. This was done both for ethical reasons and in an attempt to be close to a real clinical situation, where NSAIDs, combined with opioid drugs, are routinely used for 7 to 10 days after orthopaedic surgeries to control pain and inflammation. This is relevant because it is well known that NSAIDS inhibit cyclooxygenase-2 function, impairing bone formation at early stages ${ }^{27}$. Moreover, it has been 
suggested that a certain degree of inflammation might foster osteoinduction, through the local release of inflammatory cytokines, produced by monocytes and macrophages, which may stimulate circulating stem cells to differentiate into the osteoblastic lineage $\mathrm{e}^{28-31}$. For this reason, most studies on biomaterial-associated osteoinduction just used opioid drugs post-operatively ${ }^{12,13,26,32,33}$ or no pain-reliever/analgesic drugs $\mathrm{s}^{9,11,34-38}$.

Interestingly, the use of NSAIDs did not impair nor reduce the incidence of ectopic bone formation compared to other studies. Although it is difficult to make direct comparisons due to the various animal models and different characteristics of the materials (granules, cement pastes, blocks, etc.) we found similar or even larger amounts of ectopic bone than in previous studies with analogous designs (intramuscular implantation in large species) 9,11,12,23,26,32-34,36,38-42.

\subsection{Effect of macropore geometry on osteoinduction}

The presence of interconnected macropores is considered to be a prerequisite for osteoinduction by biomaterials ${ }^{1,12,35,43}$. This is associated to the need of body fluid flow and vascular infiltration, with the corresponding oxygen and nutrient supply, and the easy access of cells (inflammatory cells, stem cells) and soluble proteins, including signaling molecules and osteogenic growth factors ${ }^{1,11}$.

However, the results obtained in this work show that the presence of interconnected macroporosity is a necessary but not sufficient requirement. The shape of the macropores seems to be critical for osteoinduction. Three groups of CDHA scaffolds with the same chemical composition, similar percentage of interconnected macroporosity and similar nanostructure and SSA, but different macropore architecture were analysed. It was decided to keep constant the total porosity between the foamed and robocast scaffolds, and this resulted in different pore entrance sizes (Table 3). CDHA-Foam exhibited concave macropores with bottle-neck morphology, pore diameters between 10 and $300 \mu \mathrm{m}$ and pore entrance sizes around $70 \mu \mathrm{m}$. In contrast, the robocast scaffolds presented prismatic convex macropores with pore dimensions around $350 \mu \mathrm{m}$ for CDHA-Rob-450 
and $200 \mu \mathrm{m}$ for CDHA-Rob-250 (Fig. 1 and Table 3). Whereas ectopic bone was formed in the CDHA-Foam in 4 out of 6 animals at 6 weeks and in all animals at 12 weeks, in the CDHA-Rob-450 group, bone was formed just in 1 out of 6 animals at 12 weeks, in a very small amount, and no signs of bone formation were observed in the CDHA-Rob-250 at both time points (Fig. 3A/3B). Remarkably, ectopic bone in the CDHA-Rob-450 group was found only in the corners, where robocast scaffolds show concave-like regions (Fig. 5D and Fig. 6C/6F). It is worth mentioning that no correlation was found between angiogenesis and ectopic bone formation, since abundant neovascularization, homogenously distributed throughout the constructs, was observed both in the foams and in the robocast scaffolds (Fig. 2).

Although most authors recognize the positive effect of concave porosity on osteoinduction ${ }^{12,43}$, there is still controversy. Wang et al. $^{41}$ reported a better osteoinductive potential for HA-based biomaterials with channel-like architectures with convex surfaces compared to scaffolds with honeycomb-like architectures with concave surfaces. However, in our case the results pointed quite categorically to the need of concave macropores to trigger osteoinduction. This suggests that the spherical macropores provided confined spaces, which acted as niches with the adequate microenvironment for the differentiation of MSCs to osteoblasts. This includes, on one side the concentration of calcium and phosphate at the vicinity of the scaffold, and on the other the presence and retention of osteoinductive growth factors. This microenvironment would not be preserved in the more open structure of the robocast scaffolds, where both ions and proteins would easily diffuse instead of being retained locally.

Moreover, recent studies have shown that, in addition to triggering osteoinduction, geometry is a factor that contributes to controlling the kinetics of tissue deposition by osteoblastic cells ${ }^{44-46}$. Specifically, these authors have demonstrated that bone matrix deposition by osteoblasts happened in concave surfaces rather than in convex or flat surfaces, and moreover, the local growth rate of tissue formed in concave spaces was proportional to the concave curvature, which is 
in good agreement with the higher amount of bone found in the foamed scaffolds, and the localization of bone in the corners of the robocast scaffolds (Fig. 5D and Fig. 6C/6F).

\subsection{Effect of Micro/nanostructural parameters on osteoinduction}

Foamed scaffolds with similar percentage of spherical concave macroporosity but different chemical composition, microstructure and SSA were compared. The analysis of the micro-CT images, together with the BS-SEM images allowed to precisely calculating the volume of newly formed bone in each scaffold ${ }^{21}$. CDHA-Foam was the only group that showed ectopic bone formation at 6 weeks, while no signs of bone formation were observed in the sintered ceramics (BCP and $\beta$-TCP foams) at this time point. Moreover, it was the only group showing a significant amount of ectopic bone formation in all animals at 12 weeks, whilst at this time point, bone formation was observed only in 4 out of 6 animals in the BCP-Foam group and 1 out of 6 animals in the $\beta$-TCP-Foam group.

These results point in the same direction as previous studies which clearly showed that the microstructure of CaP-based biomaterials plays a determining role in stimulating osteoinduction ${ }^{2,10-}$ 13,33,42,47-49. Several in vivo studies have evaluated CaP-based biomaterials with different microstructures, consistently showing the best results in terms of osteoinduction in the CaPs with higher levels of microporosity and higher SSA ${ }^{12,13,26,32,33,42,48,49}$. However, only sintered ceramics, which have the limitation of lacking nanoporosity, have been analysed in this respect so far. Although different strategies have been explored to overcome this limitation, such as reducing the sintering temperature ${ }^{26,33,42}$, using spark plasma sintering to avoid grain coarsening ${ }^{50,51}$, or covering the surface of sintered ceramics with nano-hydroxyapatite coatings obtained by hydrothermal routes $^{52}$, these strategies did not manage to increase substantially the SSA of the materials, reaching values only around $6-10 \mathrm{~m}^{2} / \mathrm{g}$. In contrast, the biomimetic processing used in this work allowed obtaining foamed CDHA scaffolds exhibiting, in addition to the interconnected macropores, also porosity in the micro and the nanoscale, and a SSA as high as $38 \mathrm{~m}^{2} / \mathrm{g}$. 
The results obtained suggest that some mechanisms relevant for osteoinduction, such as the release of calcium and phosphate ions, in combination with the entrapment or attraction of relevant proteins and growth factors (e.g. BMPs) $)^{7,12}$ are promoted in nanostructured biomimetic materials.

It is worth mentioning that poor results in terms of ectopic bone formation were obtained in previous studies with calcium phosphate cements, with similar composition to the CDHA used in the present work $^{36,53}$. However, this could be attributed to the lack of interconnected macroporosity in the cement pastes and prehardened scaffolds. In contrast, the foaming and 3D-inkjet printing techniques used in the present study, allowed combining the required interconnected macroporosity and micro/nanoporosities relevant for the osteoinductive processes.

Besides the acceleration and promotion of the process of osteoinduction, clear differences were observed in the pattern of bone formation, when comparing CDHA and BCP Foams (Fig. 5A/5B). Whereas bone was deposited on the surface of the scaffold in the BCP-Foam, resulting in the progressive filling of the macropores, in the CDHA-Foam bone replaced the pre-existing scaffold structure, meaning that bone formation was preceded by the resorption of the scaffold. This trend was confirmed by the higher degradation rate measured for the CDHA-Foams (Fig. 7), as will be discussed below.

To better understand the role that nanostructure and phase composition played in the differentiation of MSC, rMSC were cultured without adding osteogenic factors, on the surface of discs of the different materials used in the in vivo study. An overexpression of some osteogenic genes, namely BMP-2, ONN, OPN, OCN was found more pronounced on CDHA discs than in the sintered BCP and $\beta$-TCP groups, indicating that biomimetic CDHA triggered the osteogenic differentiation of rMSCs (Fig. 8). The calcium and phosphate fluctuations registered in the cell culture medium in contact with the different materials during the cell culture study were not statistically significant (Supplementary Data, Fig. S5E). Therefore, the osteogenic differentiation produced was mostly attributed to the topographical features, which have been identified as a parameter controlling 
differentiation of mesenchymal stem cells ${ }^{54,55}$. This, in fact, is in agreement with previous studies where osteogenic differentiation of rMSC cultured on CDHA or in other nanostructured substrates like octacalcium phosphate was observed ${ }^{56,57}$. Similarly, other authors reported higher osteogenic differentiation of MSCs on low-temperature nanocrystalline HA compared to sintered HA, suggesting that nanoporosity, surface roughness and nanocrystal morphology are responsible for osteoinduction of $\mathrm{MSCs}^{58,59}$.

In this study, besides the bone formed within concave macropores from 10 to $300 \mu \mathrm{m}$ of diameter in CDHA-Foam and BCP-Foam (Fig. 6A/6B), abundant extracellular calcified bone matrix was observed within the micropores $(<10 \mu \mathrm{m})$ of both groups (Fig. 6G/6H). Other authors have previously reported the presence of calcified bone matrix within micropores. In some cases it has been associated with the osteoid produced by nearby cells being able to flow and penetrate into small micropores ${ }^{49,60,61}$. In contrast, other authors claim that this calcified tissue might be produced by the invasion of the micropores by bone cells ${ }^{62,63}$, through pore-interconnections smaller than their diameter $(4-12 \mu \mathrm{m})^{64}$. Although in our case no cells were seen within the micropores, immunohistochemical staining using for example an osteocyte marker like sclerostin ${ }^{63}$ would be needed to conclusively exclude the presence of cells. Unfortunately, this was not possible since the samples were embedded in resin, which did not allow this type of immuno-histochemical investigation.

Regarding spatial distribution, bone was found only inside the foamed constructs, but not on the external surface of the cylinders. As displayed in Fig. 5A, bone was uniformly distributed within the CDHA-Foam scaffolds with no significant differences between the five concentric VOls (Fig. 3C). However, BCP-Foams consistently showed minimal bone formation in the outer VOI (VOI 5) and most of the new bone concentrated in the middle VOI (VOI 3) (Fig. 3C and Fig. 5B). This pattern for the BCP-Foams agrees with previous studies where bone induction was observed only in the centre of the implant and not in the implant periphery. This was attributed, on one side, to the fast diffusion of the released calcium and phosphate ions at the peripheral regions of the scaffolds, which prevented reaching the supersaturation required for ectopic bone formation and on the other 
side, to the shear stresses on the outer regions of the ceramic blocks that might prevent bone tissue formation ${ }^{12}$. However, in the case of CDHA-Foams, the presence of bone uniformly distributed within the entire construct, including the periphery, can be explained by the higher reactivity of the substrate, associated both with chemical composition and textural properties, which led to higher implant degradation, resulting in higher levels of calcium and phosphate release in the whole volume of the construct.

\subsection{Degradation patterns and osteoinduction}

The role of $\mathrm{CaP}$ degradation in osteoinduction is still unknown. Some authors claim that, whereas certain degree of degradation is desirable, since it provides the calcium and phosphate ions needed to stimulate MSC differentiation and foster mineralization, a too high degradation may impair osteoinduction due to the lack of a stable surface where the new bone can be deposited ${ }^{7}$. This is the argument used to explain the higher osteoinduction found in BCP ceramics compared to both the hardly soluble sintered stoichiometric HA and the more soluble $\beta$-TCP $12,26,32,35,37,47$. The analysis of the micro-CT data obtained in this work allowed quantifying in 3D the resorption of the different scaffolds, showing different patterns when looking at sintered ceramics or at biomimetic CDHA.

When comparing the foamed scaffolds, the results showed that the material presenting the highest osteoinduction, CDHA-Foam, was the one showing also the highest degradation, above that of sintered foams (BCP and $\beta$-TCP) at 6 weeks, and above the BCP-Foam, but similar to the $\beta$-TCPFoam at 12 weeks (Fig. 7). The high degradation rate of CDHA, compared particularly to the more soluble $\beta$-TCP, agrees with previous studies ${ }^{65}$ and can be explained by its specific features, i.e., the nanosized crystals and consequently high SSA, as well as its low crystallinity and lack of stoichiometry. The degradation of the BCP-Foam, which presented a high amount of ectopic bone formation at 12 weeks, was significantly smaller. This can be associated to the low solubility of sintered HA, together with the low SSA, and can be the reason for the delayed ectopic bone formation compared to the CDHA-Foam. In the BCP-Foam, a longer time was probably required to 
achieve the supersaturation of the local environment with calcium and phosphate ions, which precedes new ectopic bone formation. On the other hand, the bone formation pattern described in the previous section is in good agreement with this lower degradation of the BCP-Foam, as bone was deposited on the surface of the scaffold instead of replacing the biomaterial (Fig. 5).

The results obtained demonstrate that the high resorption rate of CDHA-Foam, far from being an obstacle for osteoinduction, clearly promoted it. Considering that the physiological fluid is supersaturated with respect to CDHA, the homogeneous and progressive degradation of the CDHA-Foam scaffolds suggested that the high degradation profile of the CDHA-Foam scaffolds was mainly due to the multinucleated osteoclast-like cells activity (Fig. 6G), as also reported by Yuan et al. ${ }^{36}$. These results are in good agreement with previous studies, which emphasized the promotion of osteoclastic activity by submicrometric microstructures of $\mathrm{CaP}$, and its close relation with osteoinduction ${ }^{13,25,33,48,66}$. The secretion of BMPs and other osteogenic growth factors by osteoclasts are able to trigger MSCs differentiation toward the osteogenic lineage, this leading eventually to bone formation through intramembranous ossification ${ }^{7,34,66-69}$, as observed in the present study. It is noteworthy, however, that previous studies reported a lack of osteoinductive properties of carbonated hydroxyapatites with a SSA between 7 and $10 \mathrm{~m}^{2} / \mathrm{g}^{26,70}$. Therefore, it was hypothesized that there was a limit in the increase of SSA of biomaterials that positively influences osteoinduction, adducing that materials with a SSA above the optimum might degrade too fast ${ }^{26}$. In contrast, the present results demonstrate that it is possible to couple degradation and bone formation in an ectopic site.

The situation with the $\beta$-TCP foams was different. Although the degradation percentage at 12 weeks was similar to CDHA foams, the degradation pattern was very heterogeneous, leaving big holes within the constructs (Fig. 7B). The loss of a stable three-dimensional macrostructure, which is required to facilitate new bone growth was probably the main reason why this group did not show a significant ectopic bone formation (Fig. 4A/4B), despite having the same macroporosity, microstructure and SSA as the BCP-Foam group (Fig. 1 and Table 2). 
Remarkably, pore architecture also had a significant effect on the degradation of the scaffolds. CDHA-Foam scaffolds showed a higher degradation than the biomimetic robocast scaffolds at both time points (Fig. 8), in spite of having identical chemical composition, and very similar nanostructure and SSA, which should entail similar dissolution rates. Noteworthy, a higher number of osteoclast-like cells were identified in the concave macropores of the CDHA-Foam, compared to the convex prismatic macropores of the robocast scaffolds. These findings suggested that osteoclast-like cells activity, and consequently cell-mediated degradation, were enhanced by the microenvironment created inside the concave macropores. Likewise, the lack of osteoinduction by the robocast scaffolds confirms in turn the close relation between osteoclastic activity and osteoinduction.

Overall, besides providing additional information about the role of structural parameters in osteoinduction and the biological mechanisms behind it, the enhanced osteoinduction of the biomimetic CDHA-Foams has a direct clinical application for the repair of large bone defects. Some studies have demonstrated the clinical utility of osteoinductive bone substitutes for the treatment of critical sized bone defects, showing that these materials are better bone void fillers than those that are merely osteoconductive $e^{8,9,37,70}$. Thus, the next step will be to confirm this hypothesis evaluating these novel biomimetic CaP scaffolds in an orthotopic model.

\section{Conclusions}

The results obtained in this study show that both pore architecture and reactivity of the substrate play a crucial role in osteoinduction. The high reactivity of biomimetic CDHA, which results from its poor crystallinity, nanostructured nature and high SSA, combined with the concave macroporosity produced by a foaming process, resulted in an accelerated osteoinduction, when compared to conventional sintered BCP ceramics with the same macropore architecture. Different bone formation patterns were exhibited by sintered and biomimetic scaffolds. Whereas CDHA scaffolds were progressively resorbed and replaced by new ectopic bone, in the BCP scaffolds bone was 
deposited on the surface of the material, progressively filling the pore space. Conversely, very limited osteoinduction was found in the 3D robocast scaffolds with prismatic pore geometries. In conclusion, we have shown that tailoring both nanostructure and macropore geometry of biomimetic CDHA obtained by a low-temperature self-setting reaction allows pushing the osteoinduction potential beyond the limits obtained for the microstructured calcium phosphate ceramics.

\section{Associated Content}

\section{Supporting Information (PDF)}

Supporting Information is available free of charge on the ACS Publications website at DOI: XXXX, including further characterization of materials, such as XRD patterns of implanted scaffolds and BCP foams, as well as complete physicochemical characterization of planar discs used in the in vitro study. Moreover, additional results of the in vivo study, such as ectopic bone distribution in CDHA-Foam scaffolds at 6 weeks and backscattered electron micrographs of CDHA-Rob-250 scaffolds at 12 weeks of implantation are provided.

\section{Author Information}

Corresponding Author

*E-mail: maria.pau.ginebra@upc.edu

Present Address

${ }^{1}$ E.B.M.: CEITEC - Central European Institute of Technology, Brno University of Technology, 61200 Brno, Czech Republic.

Notes

The authors declare no competing financial interest.

\section{Acknowledgments}

Authors acknowledge the Spanish Government for financial support through the MAT2015-65601-R Project, co-funded by the EU through European Regional Development Funds, and the Swedish Foundation for International Cooperation in Research and Higher Education (STINT, GA IG20112047). AB acknowledges a FPU scholarship from the Spanish Ministry of Education and support for 
the research of MPG was received through the ICREA Academia award for excellence in research funded by the Generalitat de Catalunya. Authors are grateful to Dr. Cristina Costa and Dr. Pedro Fontecha from the Veterinary School at Universitat Autònoma de Barcelona for their assistance with surgical procedures, Eva Sanchez from Pathology and Experimental Therapeutics Department at Universitat de Barcelona for her technical assistance with histology and Dr. Trifon Trifonov from Barcelona Research Center in Multiscale Science and Engineering at Universitat Politècnica de Catalunya for his technical assistance with BS-SEM analysis.

\section{References}

(1) Ripamonti, U. The Morphogenesis of Bone in Replicas of Porous Hydroxyapatite Obtained from Conversion of Calcium Carbonate Exoskeletons of Coral. J. Bone Joint Surg. Am. 1991, 73, 692-703.

(2) Yamasaki, H.; Saki H. Osteogenic Response to Porous Hydroxyapatite Ceramics under the Skin of Dogs. Biomaterials 1992, 13, 308-312.

(3) Shields, L. B.; Raque, G. H.; Glassman, S. D.; Campbell, M.; Vitaz, T.; Harpring, J.; Shields, C. B. Adverse Effects Associated with High-Dose Recombinant Human Bone Morphogenetic Protein-2 Use in Anterior Cervical Spine Fusion. Spine (Phila Pa 1976) 2006, 31, 542-547.

(4) Zara, J. N.; Siu, R. K.; Zhang, X.; Shen, J.; Ngo, R.; Lee, M.; Li, W.; Chiang, M.; Chung, J.; Kwak, J.; Wu, B. M.; Ting, K.; Soo, C. High Doses of Bone Morphogenetic Protein 2 Induce Structurally Abnormal Bone And Inflammation in Vivo. Tissue Eng. Part A 2011, 17, 13891399.

(5) Giannoudis, P. V.; Dinopoulos, H.; Tsiridis, E. Bone Substitutes: an Update. Injury 2005, 36, 20-27.

(6) Younger, E. M.; Chapman, M. W. Morbidity at Bone Graft Donor Sites. J. Orthop. Trauma. 1989, 3, 92-95.

(7) Barradas, A. M. C.; Yuan, H.; van Blitterswijk, C. A.; Habibovic, P. Osteoinductive Biomaterials: Current Knowledge of Properties, Experimental Models and Biological Mechanisms. Eur. Cell. Mater. 2011, 21, 407-429. 
(8) Habibovic, P.; Yuan, H.; van den Doel, M.; Sees, T. M.; van Blitterswijk, C. A.; de Groot, K. Relevance of Osteoinductive Biomaterials in Critical-Sized Orthotopic Defect. J. Orthop. Res. 2006, 24, 867-876.

(9) Yuan, H.; Fernandes, H.; Habibovic, P.; de Boer, J.; Barradas, A. M. C.; de Ruiter, A.; Walsh, W. R.; van Blitterswijk, C. A.; de Bruijn, J. D. Osteoinductive Ceramics as a Synthetic Alternative to Autologous Bone Grafting. Proc. Natl. Acad. Sci. U.S.A. 2010, 107, 1361413619.

(10) Yuan, H.; Kurashina, K.; de Bruijn, J. D.; Li, Y.; de Groot, K.; Zhang, X. A Preliminary Study on Osteoinduction of Two Kinds of Calcium Phosphate Ceramics. Biomaterials 1999, 20, 17991806.

(11) Le Nihouannen, D.; Daculsi, G.; Saffarzadeh, A.; Gauthier, O.; Delplace, S.; Pilet, P.; Layrolle, P. Ectopic Bone Formation by Microporous Calcium Phosphate Ceramic Particles in Sheep Muscles. Bone 2005, 36, 1086-1093.

(12) Habibovic, P.; Yuan, H.; van der Valk, C. M.; Meijer, G.; van Blitterswijk, C. A.; de Groot, K. 3D Microenvironment as Essential Element for Osteoinduction by Biomaterials. Biomaterials 2005, 26, 3565-3575.

(13) Davison, N. L.; Su, J.; Yuan, H.; van den Beucken, J. J.; de Bruijn, J. D.; Barrère-de Groot, F. Influence of Surface Microstructure and Chemistry on Osteoinduction and Osteoclastogenesis by Biphasic Calcium Phosphate Discs. Eur. Cell. Mater. 2015, 29, 314329.

(14) Ginebra, M. P.; Espanol, M.; Montufar, E. B.; Perez, R. A.; Mestres, G. New Processing Approaches in Calcium Phosphate Cements and their Applications in Regenerative Medicine. Acta Biomater. 2010, 6, 2863-2873.

(15) Montufar, E. B.; Traykova, T.; Gil, C.; Harr, I.; Almirall, A.; Aguirre, A.; Engel, E.; Planell, J. A.; Ginebra, M. P. Foamed Surfactant Solution as a Template for Self-Setting Injectable Hydroxyapatite Scaffolds for Bone Regeneration. Acta Biomater. 2010, 6, 876-885.

(16) Pastorino, D.; Canal, C.; Ginebra, M. P. Drug Delivery from Injectable Calcium Phosphate Foams by Tailoring the Macroporosity-Drug Interaction. Acta Biomater. 2015, 12, 250-259. 
(17) Maazouz, Y.; Montufar, E. B.; Malbert, J.; Espanol, M.; Ginebra, M. P. Self-hardening and thermoresponsive alpha tricalcium phosphate/pluronic pastes. Acta Biomater. 2017, 49, 563574.

(18) Maazouz, Y.; Montufar, E. B.; Guillem-Marti, J.; Fleps, I.; Öhman, C.; Persson, C.; Ginebra, M. P. Robocasting of Biomimetic Hydroxyapatite Scaffolds Using Self-Setting Inks. J. Mater. Chem. B 2014, 2, 5378-5386.

(19) National Research Council. Guide for the Care and Use of Laboratory Animals. Washington, DC: National Academy Press; 1996, 41-194.

(20) Directive 2010/63/EU of the European Parliament and of the Council of 22 September 2010 on the Protection of Animals Used for Scientific Purposes.

(21) Lewin, S.; Barba, A.; Persson, C.; Franch, J.; Ginebra, M. P.; Öhman-Mägi, C. Evaluation of Bone Formation in Calcium Phosphate Scaffolds with mCT - Method Validation Using SEM. Biomed. Mater. 2017, 12, 65005.

(22) González-Vázquez, A.; Planel, J. A.; Engel, E. Extracellular Calcium and CaSR Drive Osteoinduction in Mesenchymal Stromal Cells. Acta Biomater. 2014, 6, 2824-2833.

(23) Ripamonti, U. Osteoinduction in Porous Hydroxyapatite Implanted in Heterotopic Sites of Different Animal Models. Biomaterials 1996, 17, 31-35.

(24) Yang, Z.; Yuan, H.; Tong, W.; Zou, P.; Chen, W.; Zhang, X. Osteogenesis in Extraskeletally Implanted Porous Calcium Phosphate Ceramics: Variability Among Different Kinds of Animals. Biomaterials 1996, 17, 2131-2137.

(25) Akiyama, N.; Takemoto, M.; Fujibayashi, S.; Neo, M.; Hirano, M.; Nakamura, T. Difference between Dogs and Rats with Regard to Osteoclast-Like Cells in Calcium-Deficient Hydroxyapatite-Induced Osteoinduction. J. Biomed. Mater. Res. A 2011, 96, 402-412.

(26) Habibovic, P.; Sees, T. M.; van den Doel, M. A.; van Blitterswijk, C. A.; de Groot, K. Osteoinduction by Biomaterials--Physicochemical and Structural Influences. J. Biomed. Mater. Res. A 2006, 77, 747-762.

(27) Simon, A. M.; O'Connor, J. P. Dose and Time-Dependent Effects of Cyclooxygenase-2 Inhibition on Fracture-Healing. J. Bone Joint Surg. Am. 2007, 89, 500-511. 
(28) Brown, B. N.; Ratner, B. D.; Goodman, S. B.; Amar, S.; Badylak, S. F. Macrophage

Polarization: an Opportunity for Improved Outcomes in Biomaterials and Regenerative Medicine. Biomaterials 2012, 33, 3792-3802.

(29) Mountziaris, P. M.; Spicer, P. P.; Kasper, F. K.; Mikos, A. G. Harnessing and Modulating Inflammation in Strategies for Bone Regeneration. Tissue Eng. Part B Rev. 2011, 17, 393-402.

(30) Almeida, C. R.; Vasconcelos, D. P.; Gonçalves, R. M.; Barbosa, M. A. Enhanced Mesenchymal Stromal Cell Recruitment Via Natural Killer Cells by Incorporation of Inflammatory Signals in Biomaterials. J. R. Soc. Interface 2012, 9, 261-271.

(31) Fellah, B.; Josselin, N.; Chappard, D.; Weiss, P.; Layrolle, P. Inflammatory Reaction in Rats Muscle after Implantation of Biphasic Calcium Phosphate Micro Particles. J. Mater. Sci.: Mater. Med. 2007, 18, 287-294.

(32) Habibovic, P.; Kruyt, M. C.; Juhl M. V.; Clyens, S.; Martinetti, R.; Dolcini, L.; Theilgaard, N.; Van Blitterswijk, C. A. Comparative in Vivo Study of Six Hydroxyapatite-Based Bone Graft Substitutes. J. Orthop. Res. 2008, 26, 1363-1370.

(33) Davison, N. L.; Luo, X.; Schoenmaker, T.; Everts, V.; Yuan, H.; Barrère-de Groot, F.; de Bruijn, J. D. Submicron-Scale Surface Architecture of Tricalcium Phosphate Directs Osteogenesis in Vitro and in Vivo. Eur. Cell. Mater. 2014, 27, 281-297.

(34) Kondo, N.; Ogose, A.; Tokunaga, K.; Umezu, H.; Arai, K.; Kudo, N.; Hoshino, M.; Inoue, H.; Irie, H.; Kuroda, K.; Mera, H.; Endo, N. Osteoinduction with Highly Purified Beta-Tricalcium Phosphate in Dog Dorsal Muscles and the Proliferation of Osteoclasts before Heterotopic Bone Formation. Biomaterials 2006, 27, 4419-4427.

(35) Kurashina, K.; Kurita, H.; Wu, Q.; Ohtsuka, A.; Kobayashi, H. Ectopic Osteogenesis with Biphasic Ceramics of Hydroxyapatite and Tricalcium Phosphate in Rabbits. Biomaterials 2002, 23, 407-412.

(36) Yuan, H.; Li, Y.; de Bruijn, J. D.; de Groot, K.; Zhang, X. Tissue Responses of Calcium Phosphate Cement: A Study in Dogs. Biomaterials 2000, 21, 1283-1290. 
(37) Yuan, H.; van Blitterswijk, C. A.; de Groot, K.; de Bruijn, J. D. A Comparison of Bone Formation in Biphasic Calcium Phosphate (BCP) and Hydroxyapatite (HA) Implanted in Muscle and Bone of Dogs at Different Time Periods. J. Biomed. Mater. Res. A 2006, 78, 139-147.

(38) Wang, L.; Zhang, B.; Bao, C.; Habibovic, P.; Hu, J.; Zhang, X. Ectopic Osteoid and Bone Formation by Three Calcium-Phosphate Ceramics in Rats, Rabbits and Dogs. PLoS One 2014, 9, e107044.

(39) Davison, N. L.; Yuan, H.; de Bruijn, J. D.; Barrere-de Groot, F. In Vivo Performance of Microstructured Calcium Phosphate Formulated in Novel Water-Free Carriers. Acta Biomater. 2012, 8, 2759-2769.

(40) Habibovic, P.; Gbureck, U.; Doillon, C. J.; Bassett, D. C.; van Blitterswijk, C. A.; Barralet, J. E. Osteoconduction and Osteoinduction of Low-Temperature 3D Printed Bioceramic Implants. Biomaterials 2008, 29, 944-953.

(41) Wang, H.; Zhi, W.; Lu, X.; Li, X.; Duan, K.; Duan, R.; Mu, Y.; Weng, J. Comparative Studies on Ectopic Bone Formation in Porous Hydroxyapatite Scaffolds with Complementary Pore Structures. Acta Biomater. 2013, 9, 8413-8421.

(42) Zhang, J.; Luo, X.; Barbieri, D.; Barradas, A. M. C.; de Bruijn, J. D.; van Blitterswijk, C. A.; Yuan, H. The Size of Surface Microstructures as an Osteogenic Factor in Calcium Phosphate Ceramics. Acta Biomater. 2014, 10, 3254-3263.

(43) Ripamonti, U.; Richter, P. W.; Thomas, M. E. Self-Inducing Shape Memory Geometric Cues Embedded within Smart Hydroxyapatite-Based Biomimetic Matrices. Plast. Reconstr. Surg. 2007, 120, 1796-1807.

(44) Rumpler, M.; Woesz, A.; Dunlop, J. W.; van Dongen, J. T.; Fratzl, P. The Effect of Geometry on Three-Dimensional Tissue Growth. J. R. Soc. Interface 2008, 5, 1173-1180.

(45) Bidan, C. M.; Kommareddy, K. P.; Rumpler, M.; Kollmannsberger, P.; Bréchet, Y. J.; Fratzl, P.; Dunlop, J. W. How Linear Tension Converts to Curvature: Geometric Control of Bone Tissue Growth. PLoS One 2012, 7, e36336. 
(46) Bidan, C. M.; Kommareddy, K. P.; Rumpler, M.; Kollmannsberger, P.; Fratzl, P.; Dunlop, J. W. Geometry as a Factor for Tissue Growth: Towards Shape Optimization of Tissue Engineering Scaffolds. Adv. Healthc. Mater. 2013, 2, 186-194.

(47) Yuan, H.; van den Doel, M.; Li, S.; Van Blitterswijk, C. A.; De Groot, K.; De Bruijn, J. D. A Comparison of the Osteoinductive Potential of Two Calcium Phosphate Ceramics Implanted Intramuscularly in Goats. J. Mater. Sci.: Mater. Med. 2002, 13, 1271-1275.

(48) Davison, N. L.; Gamblin, A-L.; Layrolle, P.; Yuan, H.; de Bruijn, J. D.; Barrère-de Groot, F. Liposomal Clodronate Inhibition of Osteoclastogenesis and Osteoinduction by Submicrostructured Beta-Tricalcium Phosphate. Biomaterials 2014, 35, 5088-5097.

(49) Chan, O.; Coathup, M. J.; Nesbitt, A.; Ho, C. Y.; Hing, K. A.; Buckland, T.; Campion, C.; Blunn, G.W. The Effects of Microporosity on Osteoinduction of Calcium Phosphate Bone Graft Substitute Biomaterials. Acta Biomater. 2012, 8, 2788-2794.

(50) Guo, X. Y.; Gough, J. E.; Xiao, P.; Liu, J.; Shen, Z. J. Fabrication of Nanostructured Hydroxyapatite and Analysis of Human Osteoblastic Cellular Response. J. Biomed. Mater. Res. A 2007, 82, 1022-1032.

(51) Li, B.; Chen, X. N.; Guo, B.; Wang, X. L.; Fan, H. S.; Zhang, X. D. Fabrication and Cellular Biocompatibility of Porous Carbonated Biphasic Calcium Phosphate Ceramics with a Nanostructure. Acta Biomater. 2009, 5, 134-143.

(52) Hu, J.; Zhou, Y.; Huang, L.; Liu, J.; Lu, H. Effect of Nano-Hydroxyapatite Coating on the Osteoinductivity of Porous Biphasic Calcium Phosphate Ceramics. BMC Musculoskelet. Disord. 2014, 15, 114.

(53) Gosain, A. K.; Song, L.; Riordan, P.; Amarante, M. T.; Nagy, P. G.; Wilson, C. R.; Toth, J. M.; Ricci, J. L. A 1-Year Study of Osteoinduction in Hydroxyapatite-Derived Biomaterials in an Adult Sheep Model: Part I. Plast. Reconstr. Surg. 2002, 109, 619-630.

(54) McBeath, R.; Pirone, D. M.; Nelson, C. M.; Bhadriraju, K.; Chen, C. S. Cell Shape, Cytoskeletal Tension, and RhoA Regulate Stem Cell Lineage Commitment. Dev. Cell 2004, 6, 483-495. 
(55) Kilian, K. A.; Bugarija, B.; Lahn, B. T.; Mrksich, M. Geometric Cues for Directing the Differentiation of Mesenchymal Stem Cells. Proc. Natl. Acad. Sci. U.S.A. 2010, 107, 48724877.

(56) Sadowska, J. M.; Guillem-Marti, J.; Montufar, E. B.; Espanol, M.; Ginebra, M. P. Biomimetic Versus Sintered Calcium Phosphates: The in Vitro Behavior of Osteoblasts and Mesenchymal Stem Cells. Tissue Eng. Part $A$ 2017. [ahead of print: https://doi.org/10.1089/ten.tea.2016.0406]

(57) Danoux, C.; Pereira, D.; Döbelin, N.; Stähli, C.; Barralet, J.; van Blitterswijk, C.; Habibovic, P. The Effects of Crystal Phase and Particle Morphology of Calcium Phosphates on Proliferation and Differentiation of Human Mesenchymal Stromal Cells. Adv. Healthc. Mater. 2016, 5, 1775-1785.

(58) Hesaraki, S.; Nazarian, H.; Pourbaghi-Masouleh, M.; Borhan, S. Comparative Study of Mesenchymal Stem Cells Osteogenic Differentiation on Low-Temperature Biomineralized Nanocrystalline Carbonated Hydroxyapatite and Sintered Hydroxyapatite. J. Biomed. Mater. Res. B Appl. Biomater. 2014, 102, 108-118.

(59) Nagai, H.; Kobayashi-Fujioka, M.; Fujisawa, K.; Ohe, G.; Takamaru, N.; Hara, K.; Uchida, D.; Tamatani, T.; Ishikawa, K.; Miyamoto, Y. Effects of Low Crystalline Carbonate Apatite on Proliferation and Osteoblastic Differentiation of Human Bone Marrow Cells. J. Mater. Sci. Mater. Med. 2015, 26, 99.

(60) Dellinger, J. G.; Eurell, J. A. C.; Jamison, R. D. Bone Response to 3D Periodic Hydroxyapatite Scaffolds with and without Tailored Microporosity to Deliver Bone Morphogenetic Protein 2. J. Biomed. Mater. Res. A 2005, 76, 366-376.

(61) Simon, J. L; Michna, S.; Lewis, J. A.; Rekow, E. D.; Thompson, V. P.; Smay, J. E.; Yampolsky, A.; Parsons, J. R.; Ricci, J. L. In Vivo Bone Response to 3D Periodic Hydroxyapatite Scaffolds Assembled by Direct Ink Writing. J. Biomed. Mater. Res. A 2007, 83, 747-758.

(62) Lapczyna, H.; Galea, L.; Wüst, S.; Bohner, M.; Jerban, S.; Sweedy, A.; Doebelin, N.; van Garderen, N.; Hofmann, S.; Baroud, G.; Müller, R.; von Rechenberg, B. Effect of Grain Size 
And Microporosity on the in Vivo Behaviour of B-Tricalcium Phosphate Scaffolds. Eur. Cell. Mater. 2014, 28, 299-319.

(63) Bohner, M.; Baroud, G.; Bernstein, A.; Döbelin, N.; Galea, L.; Hesse, B.; Heuberger, R.; Meille, S.; Pascal, M.; von Rechenberg, B.; Sague, J.; Seeherman, H. Characterization and Distribution of Mechanically Competent Mineralized Tissue in Micropores of B-Tricalcium Phosphate Bone Substitutes. Mater. Today 2017, 20, 106-115.

(64) Polak, S. J.; Rustom, L. E.; Genin, G. M.; Talcott, M.; Wagoner-Johnson, A. J. A Mechanism for Effective Cell-Seeding in Rigid, Microporous Substrates. Acta Biomater. 2013, 9, 79777986.

(65) Díez-Escudero, A.; Espanol, M.; Beats, S.; Ginebra, M. P. In Vitro Degradation of Calcium Phosphates: Effect of Multiscale Porosity, Textural Properties and Composition. Acta Biomater. 2017, 60, 81-92.

(66) Ripamonti, U.; Klar, R. M.; Renton, L. F.; Ferretti, C. Synergistic Induction of Bone Formation by Hop-1, Htgf-Beta3 and Inhibition by Zoledronate in Macroporous Coral-Derived Hydroxyapatites. Biomaterials 2010, 31, 6400-6410.

(67) Garimella, R.; Tague, S. E.; Zhang, J.; Belibi, F.; Nahar, N.; Sun, B. H.; Insogna, K.; Wang, J.; Anderson, H. C. Expression and Synthesis of Bone Morphogenetic Proteins by Osteoclasts: A Possible Path to Anabolic Bone Remodeling. J. Histochem. Cytochem. 2008, $56,569-577$.

(68) Pederson, L.; Ruan, M.; Westendorf, J.J.; Khosla, S.; Oursler, M. J. Regulation of Bone Formation by Osteoclasts Involves Wnt/BMP Signaling and the Chemokine Sphingosine-1Phosphate. Proc. Natl. Acad. Sci. U.S.A. 2008, 105, 20764-20769.

(69) Klar, R. M.; Duarte, R.; Dix-Peek, T.; Dickens, C.; Ferretti, C.; Ripamonti, U. Calcium lons and Osteoclastogenesis Initiate the Induction of Bone Formation by Coral-Derived Macroporous Constructs. J. Cell. Mol. Med. 2013, 17, 1444-1457.

(70) Habibovic, P.; Juhl, M. V.; Clyens, S.; Martinetti, R.; Dolcini, L.; Theilgaard, N.; van Blitterswijk, C. A. Comparison of Two Carbonated Apatite Ceramics in Vivo. Acta Biomater. 2010, 6, 2219-2226. 
Table of Contents/Abstract Graphic
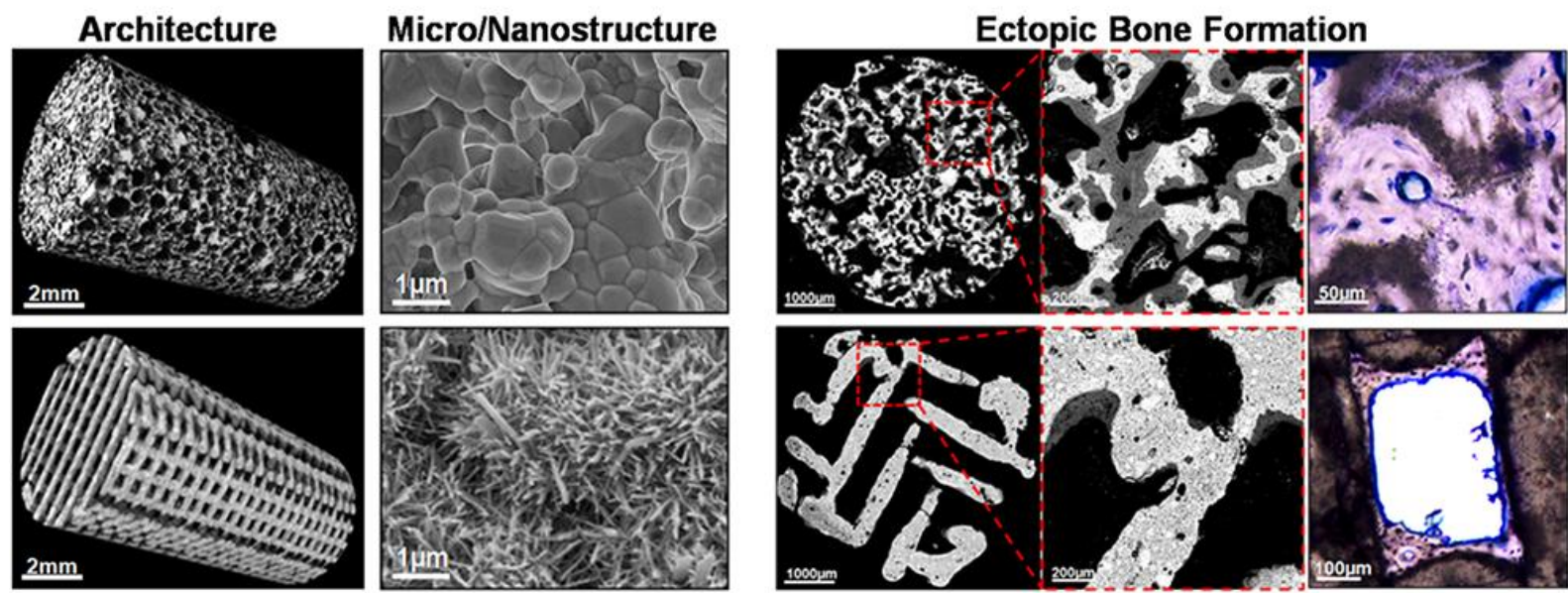